\title{
19. EOCENE TO QUATERNARY BENTHIC FORAMINIFERS AND PALEOBATHYMETRY OF THE IZU-BONIN ARC, LEGS 125 AND $126^{1}$
}

\author{
Kunio Kaiho ${ }^{2}$
}

\begin{abstract}
The benthic foraminifer fauna at Sumisu Rift Sites 790 and 791 indicates that a deep open-ocean $(>2300 \mathrm{~m})$ or a basin with open-ocean access existed between 1.1 and $0.7 \mathrm{Ma}$ at the time of the initiation of rifting. The appearance of a low- to medium-oxygen fauna $(1600-2300 \mathrm{~m})$ between 0.7 and 0.5 Ma suggests that the open-ocean access may have been terminated at this time because of the development of volcanoes and rift flank uplifts around the basin. The occurrence of low-oxygen faunas at 0.03 Ma suggests a secondary closing of the basin.

The lower bathyal benthic faunas from lower Pliocene sediments of rift margin Site 788 suggest about $0.6-1.6 \mathrm{~km}$ of total basement uplift. This uplift may have led to the formation of the major hiatus between 2.3 and $<0.3 \mathrm{Ma}$.

The faunal changes of benthic foraminifers at Sites 792 and 793 in the forearc basin document a shallowing water depth from below the carbonate compensation depth (CCD) (about $3.5 \mathrm{~km}$ ) in the late early Oligocene to the present depths of 1800 and $2975 \mathrm{~m}$, respectively. These data suggest about $1 \mathrm{~km}$ of total basement uplift in the inner part of the forearc basin (Site 792 ) and about $0.6 \mathrm{~km}$ total basement subsidence in the central part of the forearc basin (Site 793) since about $31 \mathrm{Ma}$. The former uplift led to a thinner sediment accumulation $(800 \mathrm{~m})$ and the latter subsidence to a thicker sediment accumulation $(1400 \mathrm{~m})$ at these sites.

Faunal changes of benthic foraminifers observed in Sites 782 and 786 sequences drilled at the outer-arc high document a deepening water depth from 1.3 to $2.1 \mathrm{~km}$ in late Eocene to the present depth of about $3 \mathrm{~km}$. These data suggest about 1.1-1.9 and $1.3-2.1 \mathrm{~km}$ of total basement subsidence at Sites 786 and 782 , respectively.

These results indicate total basement uplift in the inner part of the Bonin arc-trench system since late Oligocene and total basement subsidence in the outer part of the system since late Eocene.

The last occurrence (LO) of Stilostomella spp. and Pleurostomella spp. and the first occurrence (FO) of Bulimina aculeata d'Orbigny occurred consistently at $0.7 \mathrm{Ma}$ at all three arc proximal sites $(790,791$, and 792$)$. This fact is taken to suggest a change of water mass, from one originating from the central part of the ocean to that originating from ocean-margin areas at that time.
\end{abstract}

\section{INTRODUCTION}

The Ocean Drilling Program (ODP) Leg 126 Shipboard Scientific Party drilled in the Sumisu Rift to investigate geological processes associated with a renewed rifting of the arc (before backarc spreading). They also drilled in the forearc basin and discovered that it, too, was formed by rifting in the middle Oligocene (28-31 Ma) (Leg 126 Shipboard Scientific Party, 1989; Fujioka et al., 1989) (Figs. 1-2 and Table 1). Sites drilled in the outer-arc high by the Leg 125 Shipboard Scientific Party recovered middle Eocene to Holocene sediments.

At Sumisu Rift Sites 790 and 791, four cycles of explosive volcanic activity in the last $150 \mathrm{k}$.y. deposited glassy and pumiceous silts, sands, and gravels. The pumiceous unit overlies nannofossil-rich clays, with interbeds of thin ash layers and scattered pumice and scoria clasts that date back to $1.1 \mathrm{Ma}$ (Leg 126 Shipboard Scientific Party, 1989). Only about $30 \mathrm{~m}$ of pumiceous gravels were deposited during the past 275 k.y., above a major unconformity at rift margin Site 788. The unconformity is the result of margin uplift of between 200 and $1700 \mathrm{~m}$ since the deposition of the underlying pumiceous units of $2.35-3.56 \mathrm{Ma}$. The rift formed very quickly, beginning at $2 \pm 0.5 \mathrm{Ma}$ (Leg 126 Shipboard Scientific Party, 1989).

At three forearc sites (787, 792, and 793), sedimentation occurred initially at a rate of $250-300 \mathrm{~m} / \mathrm{m}$.y., and debris-flow deposits and turbidites were produced by concurrent volcanism and the erosion of surrounding topographic highs. By the early Miocene, arc volcanism had become very much reduced, as evidenced by the slow accumulation $(10 \mathrm{~m} / \mathrm{m} . \mathrm{y}$.) of claystones and nannofossil claystones (Leg 126 Shipboard Scientific Party, 1989; Egeberg et al., 1990). From 13 Ma to the present, the volcanogenic input increased and became moder-

\footnotetext{
${ }^{1}$ Taylor, B., Fujioka, K., et al., 1992. Proc. ODP, Sci. Results, 126: College Station, TX (Ocean Drilling Program).

${ }^{2}$ Institute of Geology and Paleontology, Tohoku University, Sendai 980, Japan.
}

ate. The early and early-middle Miocene minimum in volcanism corresponds in time with the backarc opening of the Shikoku Basin (Leg 126 Shipboard Scientific Party, 1989).

At outer-arc-high Sites 782 and 786, middle Eocene to Holocene sediments are mainly composed of nannofossil chalk, marl, and clay. Sedimentation rates were very low $(1.1-4.9 \mathrm{~m} / \mathrm{m} . \mathrm{y}$.) in the Eocene and Oligocene and relatively high $(4.2-16.5 \mathrm{~m} / \mathrm{m} . \mathrm{y}$.) since the middle Miocene, with two hiatuses occurring in early Oligocene and early Miocene at both sites (Fryer, Pearce, Stokking, et al., 1990).

To clarify the geological history of the Izu-Bonin Arc region, the determination of paleowater depths and vertical motion of the basement are essential factors. Benthic foraminifers worked well as a prominent instrument to estimate these data during on-board studies. The present study also reveals that a change in dissolved-oxygen conditions in the Sumisu Rift and a faunal change occurred at $0.7 \mathrm{Ma}$ in this region. I report here a benthic foraminifer biostratigraphy, paleowater depths, and dissolved-oxygen conditions and attempt to estimate the vertical motion of the basement as well as the oceanic environmental changes that took place in the Izu-Bonin Arc region.

This is the first report of smaller benthic foraminifer fossils from the Izu-Bonin region. I was able to obtain abundant, well-preserved, deep-sea benthic foraminifer faunas from all the cored sequences except for the Oligocene and Miocene sediments in the forearc Sites 792 and 793 , which contain much fewer numbers of specimens. Tables 2-3 and Figures 3-7 present the stratigraphic distribution of benthic foraminifers and their abundances. The plate illustrations (Plates 1-8) cover most of the species found in the present study. No illustrations of specimens from Leg 125 are given for the plates.

\section{MATERIALS AND METHODS}

The benthic foraminifer biostratigraphy presented in this study is based on the on-board examination of core-catcher samples and additional samples taken from each core section. I used only those 

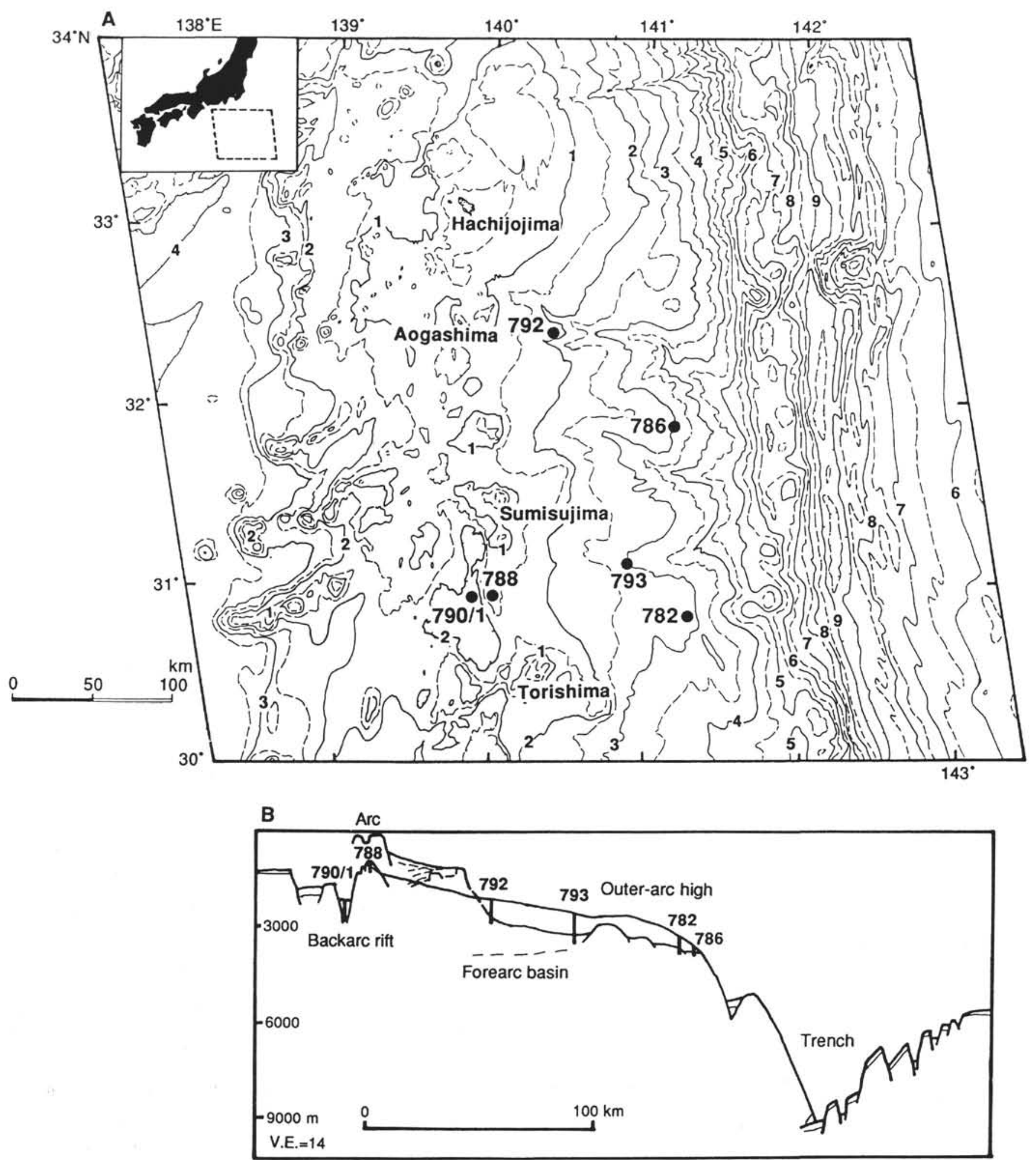

Figure 1. A. Bathymetric map (500-m contour interval) of Izu-Bonin region and locations of drill sites studied. B. Bathymetric profile in this region and locations of drill sites studied. $\mathrm{VE}=$ vertical exaggeration. 
A
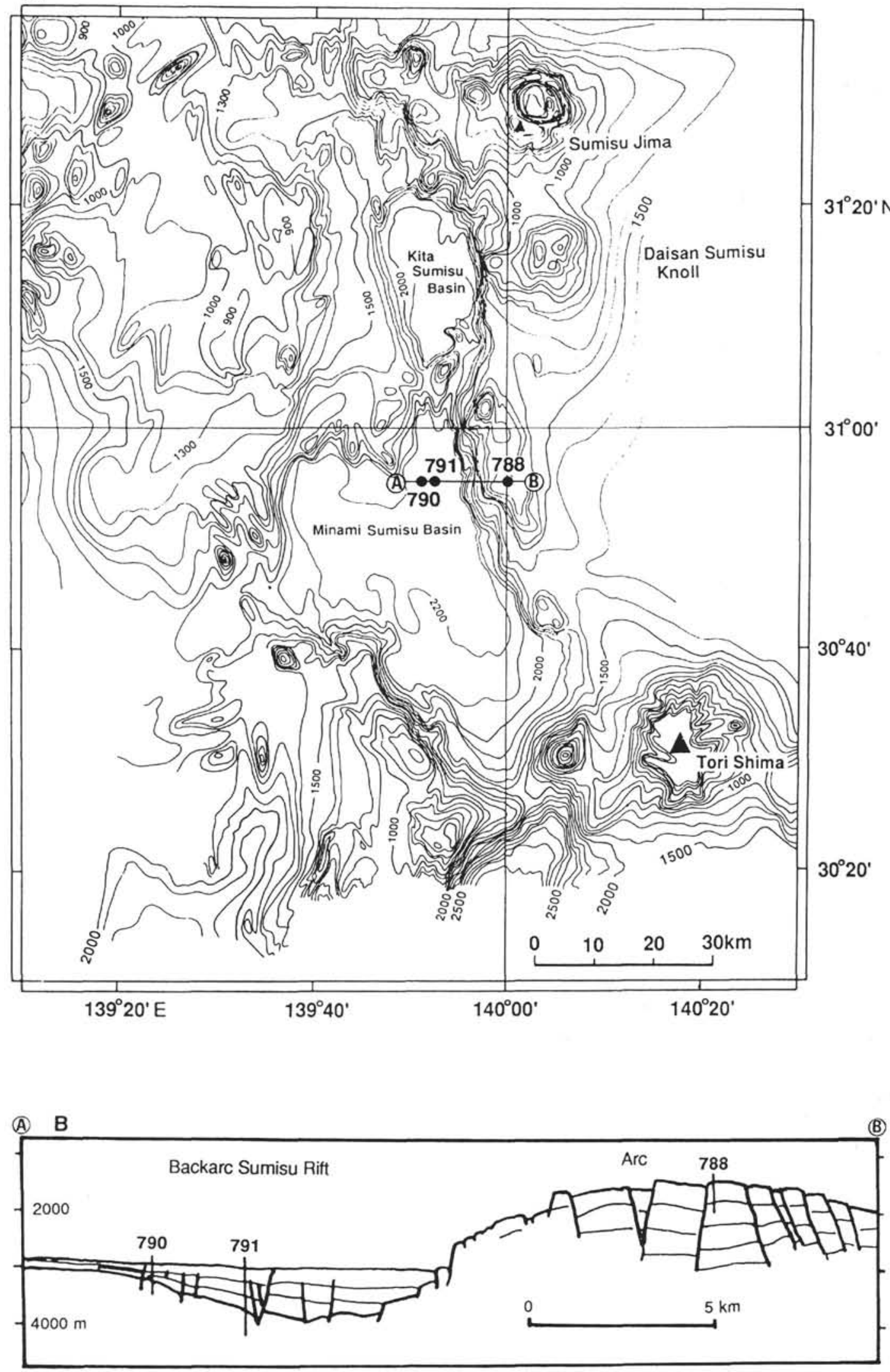

Figure 2. A. Bathymetric map (100-m contour interval) of Sumisu Rift (Murakami, 1988) and location of Sites 788, 790, and 791. B. Cross section along line of drill sites. The ends of the profile, labeled A and B, are located in the map above. 
Table 1. Locations and water depths of drill holes studied.

\begin{tabular}{ccccc}
\hline Leg & Hole & $\begin{array}{c}\text { Latitude } \\
\left({ }^{\circ} \mathrm{N}\right)\end{array}$ & $\begin{array}{c}\text { Longitude } \\
\left({ }^{\circ} \mathrm{E}\right)\end{array}$ & $\begin{array}{c}\text { Water } \\
\text { depth } \\
(\mathrm{m})\end{array}$ \\
\hline 125 & $782 \mathrm{~A}$ & $30^{\circ} 51.66^{\prime}$ & $141^{\circ} 18.85^{\prime}$ & 2958.9 \\
125 & $786 \mathrm{~A}$ & $31^{\circ} 52.48^{\prime}$ & $141^{\circ} 13.58^{\prime}$ & 3058.1 \\
126 & $788 \mathrm{C}$ & $30^{\circ} 55.36^{\prime}$, & $140^{\circ} 00.21^{\prime}$ & 1113.0 \\
126 & $788 \mathrm{D}$ & $30^{\circ} 55.37^{\prime}$ & $140^{\circ} 00.22^{\prime}$ & 1113.0 \\
126 & $790 \mathrm{~A}$ & $30^{\circ} 54.95^{\prime}$, & $139^{\circ} 50.66^{\prime}$ & 2221.7 \\
126 & $790 \mathrm{~B}$ & $30^{\circ} 54.96^{\prime}$ & $139^{\circ} 50.66^{\prime}$ & 2223.0 \\
126 & $790 \mathrm{C}$ & $30^{\circ} 54.95^{\prime}$ & $139^{\circ} 50.69^{\prime}$ & 2223.0 \\
126 & $791 \mathrm{~A}$ & $30^{\circ} 54.96^{\prime}$ & $139^{\circ} 52.20^{\prime}$ & 2268.0 \\
126 & $791 \mathrm{~B}$ & $30^{\circ} 54.98^{\prime}$ & $139^{\circ} 52.19^{\prime}$ & 2268.0 \\
126 & $792 \mathrm{~A}$ & $32^{\circ} 23.97^{\prime}$ & $140^{\circ} 22.81^{\prime}$ & 1786.8 \\
126 & $792 \mathrm{~B}$ & $32^{\circ} 23.96^{\prime}$ & $140^{\circ} 22.81^{\prime}$ & 1787.2 \\
126 & $792 \mathrm{E}$ & $32^{\circ} 23.96^{\prime}$ & $140^{\circ} 22.79^{\prime}$ & 1787.2 \\
126 & $793 \mathrm{~A}$ & $31^{\circ} 06.35^{\prime}$ & $140^{\circ} 32.26^{\prime}$ & 2964.3 \\
126 & $793 \mathrm{~B}$ & $31^{\circ} 06.33^{\prime}$ & $140^{\circ} 53.27^{\prime}$ & 2964.3 \\
\hline
\end{tabular}

Note: Water depth is given in meters below mean sea level, corrected from drill-pipe measurements from the rig floor.

samples that are mainly composed of silt and clay. Of these, about two-thirds of the samples from the Oligocene turbidite layers are barren of foraminifers, and samples from Quaternary ash beds contain very rare benthic foraminifers. I did not use the latter samples for the present analysis.

Samples of $20-30 \mathrm{~cm}^{3}$ were washed through a 250 -mesh screen (63- $\mu \mathrm{m}$ opening) and dried. Unconsolidated sediments were soaked in water, and, when necessary, disaggregation was aided with the addition of a small amount of hydrogen peroxide. Hydrogen peroxide was also routinely added to more consolidated sediments. Kerosene was used to disaggregate highly consolidated sediments.

Depositional water depths are estimated mainly on the basis of a comparison with Holocene benthic foraminifer data from the Izu-Bonin region (Kaiho and Nishimura, this volume) for the Pliocene and Pleistocene strata and on the basis of Cenozoic benthic foraminifer data (Ingle, 1980; Woodruff, 1985; van Morkhoven et al., 1986) for the Miocene and Oligocene strata. As the Holocene carbonate compensation depth (CCD) in the Izu-Bonin region was estimated to lie at about $3.7 \mathrm{~km}$ depth (Kaiho and Nishimura, this volume), and the CCD in the Oligocene was about $0.2 \mathrm{~km}$ shallower than the present in outer equatorial zones of the Pacific Ocean (van Andel et al., 1975), I used a depth of $3.5 \mathrm{~km}$ as the Oligocene $\mathrm{CCD}$ in the Izu-Bonin region in this study.

Dissolved oxygen contents were estimated from the ratios of aerobic vs. anaerobic forms of benthic foraminifers (Kaiho, 1989, in press).

The ages of the strata are based on calcareous nannofossil zonations (Taylor, Fujioka, et al., 1990; Firth and Isiminger-Kelso, this volume) and the magnetic polarity reversal record (Taylor, Fujioka, et al., 1990). A general correlation between the biostratigraphic zones and the magnetic polarity reversal record, the seafloor anomalies, and an absolute time scale is based on the scheme given by Berggren et al. (1985).

\section{BENTHIC FORAMINIFER BIOSTRATIGRAPHY, PALEOBATHYMETRY, AND PALEOENVIRONMENTS}

\section{Sumisu Rift}

The Quaternary strata at Sites 790 and 791 may be subdivided biostratigraphically into six biozonules based on benthic foraminifers (Figs. 3-5).

\section{Pleurostomella spp.-Stilostomella spp. Assemblage Zonule}

Definition: This assemblage zonule is marked by a joint occurrence of Pleurostomella spp. and Stilostomella spp.

Characteristic species: Uvigerina hispidocostata, Oridorsalis umbonatus, Cibicidoides wuellerstorfi, Stilostomella spp., Melonis barleeanus, Melonis pompilioides, Pullenia bulloides, and Pleurostomella spp.

Samples: From 126-790C-20X-CC through -18X-CC; and from 126-791B-47R-1, 41-43 cm, through -37R-CC.

Depositional water depth: $2300-3500 \mathrm{~m}$.

Dissolved oxygen: High.

Environment: A basin with at least one open passage of water.

\section{Bulimina aculeata Assemblage Zonule}

Definition: This zonule corresponds to an interval from the LO of Pleurostomella spp. to the base of the acme zonule of Uvigerina senticosa.

Characteristic species: Uvigerina hispidocostata, Bulimina aculeata, Oridorsalis umbonatus, and Melonis barleeanus.

Samples: 126-790C-17X-CC and 126-791B-36R-CC.

Depositional water depth: $1600-2300 \mathrm{~m}$.

Dissolved oxygen: Medium high.

Event: Slight shallowing or closing of the basin started; a change of water mass from the one originating from the central part of the ocean to that originating from ocean margin areas.

\section{Uvigerina senticosa Acme Zonule}

Definition: This zone corresponds to the acme zonule of Uvigerina senticosa.

Characteristic species: Hoeglundina elegans, Uvigerina senticosa, Bulimina striata, Bulimina aculeata, Melonis barleeanus, Melonis pompilioides, Pullenia bulloides, Sphaeroidina bulloides, and Oridorsalis umbonatus.

Samples: From 126-790C-15X-CC through -13X-3, 104-106 $\mathrm{cm}$, and from 126-791B-35R-CC through -25R-CC.

Depositional water depth: $1600-2300 \mathrm{~m}$.

Dissolved oxygen: Low medium and partly low; low oxygen in Samples 126-790C-14X-2, 61-63 cm, and -13X-CC and in Samples 126-791B-29R-CC and -28R-CC because of the occurrence of Globobulimina affinis and low ratios of aerobic forms.

Event: Closing of the basin probably occurred and dissolved oxygen decreased in this basin; shallower fauna entered into this basin and the faunal change occurred.

\section{Bulimina striata-Bulimina aculeata Assemblage Zonule}

Definition: This zonule represents a body of strata characterized by the joint occurrence of Bulimina striata and Bulimina aculeata.

Characteristic species: Uvigerina hispidocostata, Hoeglundina elegans, Bulimina aculeata, Oridorsalis umbonatus, Melonis barleeanus, Bulimina striata, and Melonis pompilioides.

Samples: From 126-790C-12X-CC through 126-790B-10H-CC, and from 126-791B-24R-CC through 126-791A-38X-CC.

Depositional water depth: $1600-2300 \mathrm{~m}$.

Dissolved oxygen: Medium.

Event: Closed environment still continued but dissolved oxygen increased.

\section{Bulimina aculeata-Uvigerina hispidocostata Assemblage Zonule}

Definition: The present zonule represents a body of strata characterized by the joint occurrence of Bulimina aculeata and Uvigerina hispidocostata.

Characteristic species: Uvigerina hispidocostata, Melonis barleeanus, Oridorsalis umbonatus, Bulimina aculeata, Pullenia bulloides, Pyrgo murrhina, and Tosaia hanzawai.

Samples: From 126-790B-8H-6, 29-31 cm, through -7H-CC, and from 126-791A-33X-1, 6-8 cm, through 126-791A-16H-6, 58-60 cm.

Depositional water depth: $1600-2300 \mathrm{~m}$.

Dissolved oxygen: Medium. 
Table 2. List of samples from Leg 126 studied in this report.

\begin{tabular}{|c|c|}
\hline $\begin{array}{l}\text { Core, section, } \\
\text { interval (cm) }\end{array}$ & $\begin{array}{c}\text { Sample } \\
\text { no. }\end{array}$ \\
\hline $\begin{array}{l}\text { 126-790A- } \\
1 \mathrm{H}-1,7-9 \\
2 \mathrm{H}-1,129-131 \\
2 \mathrm{H}-3,98-100\end{array}$ & $\begin{array}{l}1 \\
2 \\
3\end{array}$ \\
\hline $\begin{array}{l}126-790 \mathrm{~B}- \\
3 \mathrm{H}-1,0-2 \\
7 \mathrm{H}-\mathrm{CC} \\
8 \mathrm{HC}, 29-31 \\
10 \mathrm{H}-\mathrm{CC}\end{array}$ & $\begin{array}{l}4 \\
5 \\
6 \\
7\end{array}$ \\
\hline 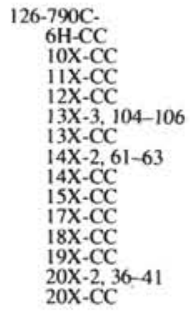 & $\begin{array}{r}8 \\
9 \\
10 \\
11 \\
12 \\
13 \\
14 \\
15 \\
16 \\
17 \\
18 \\
19 \\
20 \\
21\end{array}$ \\
\hline $\begin{array}{l}\text { 126-791A- } \\
4 \mathrm{H}-3,60-62 \\
5 \mathrm{H}-3,52-54 \\
16 \mathrm{H}-6,58-60 \\
16 \mathrm{H}-\mathrm{CC}-60 \\
18 \mathrm{H}-1,15-21 \\
22 \mathrm{H}-6,66-68 \\
25 \mathrm{X}-1,5-10 \\
31 \mathrm{X}-\mathrm{CC} \\
33 \mathrm{X}-1.6-8 \\
38 \mathrm{X}-\mathrm{CC} \\
39 \mathrm{X}-1,28-32 \\
40 \mathrm{X}-\mathrm{CC} \\
41 \mathrm{X}-\mathrm{CC} \\
43 \mathrm{X}-\mathrm{CC} \\
46 \mathrm{X}-\mathrm{CC}\end{array}$ & $\begin{array}{r}1 \\
2 \\
3 \\
4 \\
5 \\
6 \\
7 \\
8 \\
9 \\
10 \\
11 \\
12 \\
13 \\
14 \\
15\end{array}$ \\
\hline $\begin{array}{l}126-791 B- \\
9 R-C C \\
10 R-C C \\
16 R-C C \\
19 R-C C \\
20 R-C C \\
22 R-C C \\
23 R-C C \\
24 R-C C \\
25 R-C C \\
26 R-C C \\
27 R-C C \\
28 R-C C \\
29 R-C C \\
30 R-C C \\
31 R-C C \\
34 R-C C \\
35 R-C C \\
36 R-C C \\
37 R-C C \\
38 R-C C \\
39 R-C C \\
40 R-C C \\
41 R-C C \\
43 R-C C \\
44 R-C C \\
45 R-C C \\
46 R-C C \\
47 R-1,41-43\end{array}$ & $\begin{array}{l}16 \\
17 \\
18 \\
19 \\
20 \\
21 \\
22 \\
23 \\
24 \\
25 \\
26 \\
27 \\
28 \\
29 \\
30 \\
31 \\
22 \\
33 \\
34 \\
35 \\
36 \\
37 \\
38 \\
39 \\
40 \\
41 \\
42 \\
43\end{array}$ \\
\hline $\begin{array}{l}126-792 \mathrm{~A}- \\
\text { HH-CC } \\
2 \mathrm{C}-2.57-62 \\
3 \mathrm{H}-3,59-64 \\
4 \mathrm{H}-\mathrm{CC} \\
5 \mathrm{H}-\mathrm{CC} \\
7 \mathrm{H}-1.59-61 \\
8 \mathrm{H}-1.93-97 \\
9 \mathrm{H}-\mathrm{CC}-97 \\
10 \mathrm{H}-3,62-66\end{array}$ & $\begin{array}{l}1 \\
2 \\
3 \\
4 \\
5 \\
6 \\
7 \\
8 \\
9\end{array}$ \\
\hline $\begin{array}{l}126-792 \mathrm{~B}- \\
7 \mathrm{X}-\mathrm{CC} \\
8 \mathrm{X}-3,67-72 \\
8 \mathrm{X}-\mathrm{CC} \\
9 \mathrm{X}-\mathrm{CC} \\
10 \mathrm{X}-3,52-54\end{array}$ & $\begin{array}{l}10 \\
11 \\
12 \\
13 \\
14\end{array}$ \\
\hline $\begin{array}{l}\text { 126-792E- } \\
\text { IR-1, 11-13 } \\
\text { IR-CC } \\
\text { 2R-1, 13-15 } \\
\text { 2R-CC } \\
\text { 3R-1, 64-69 } \\
\text { 3R-CC } \\
\text { 4R-1, } \\
\text { 4R-11 }\end{array}$ & $\begin{array}{l}16 \\
17 \\
18 \\
19\end{array}$ \\
\hline
\end{tabular}

Table 2 (continued).

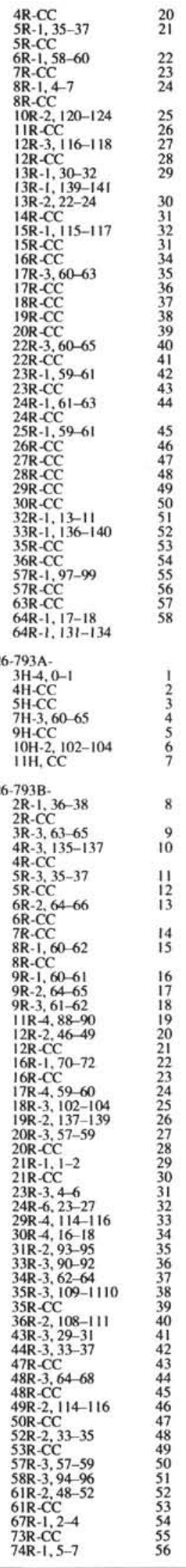


Table 3. Distribution chart of benthic foraminifers from the upper Eocene to the Pleistocene at Sites 782 and 786.

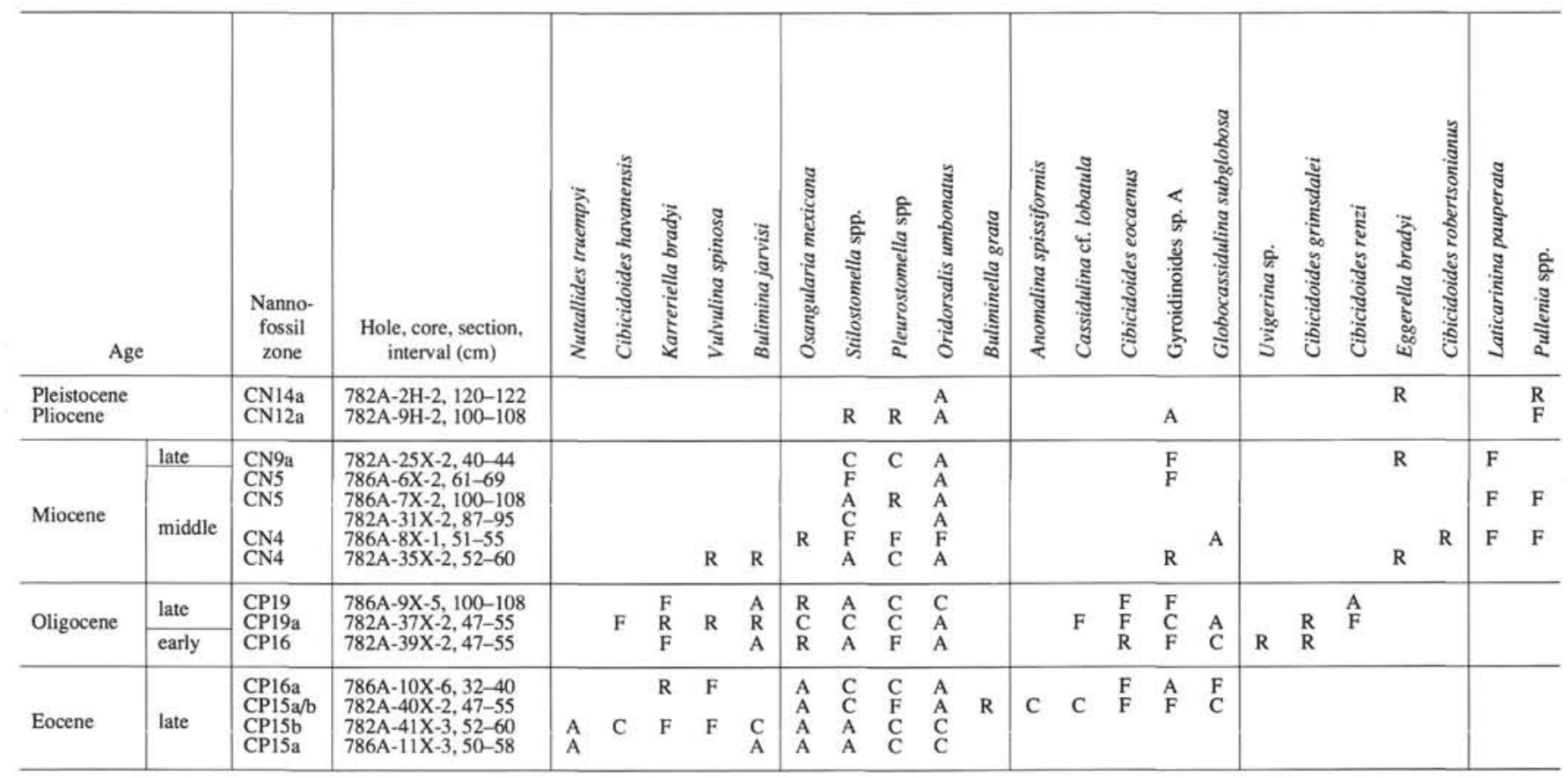

Event: Sill depth probably deepened; thick volcanic ash was deposited above the present zonule and the faunal change occurred.

\section{Chilostomella oolina-Uvigerina hispidocostata Assemblage Zonule}

Definition: The zonal base is defined by a rapid increase in the abundance of Chilostomella oolina.

Characteristic species: Chilostomella oolina, Uvigerina hispidocostata, Melonis barleeanus, and Globobulimina affinis.

Samples: From 126-790B-3H-1, 0-2 cm, through 126-790A-1H$1,7-9 \mathrm{~cm}, 126-791 \mathrm{~A}-5 \mathrm{H}-3,52-54 \mathrm{~cm}$, and $-4 \mathrm{H}-3,60-62 \mathrm{~cm}$.

Depositional water depth: $1600-2300 \mathrm{~m}$.

Dissolved oxygen: Low medium and partly low; low oxygen in Samples 126-790A-2H-3, 98-100 cm, and -2H-1, 129-131 cm, and in Samples 126-791A-5H-3, 52-54 cm, and $-4 \mathrm{H}-3,60-62 \mathrm{~cm}$, because of the occurrences of Chilostomella oolina and Globobulimina affinis and the low ratios of the aerobic forms.

Event: Anaerobic fauna increased.

\section{Rift Margin Site 788}

A benthic foraminifer fauna from Samples 126-788C-28X-CC, 5-6, 13-14, and 14-18 cm, and from Samples 126-788D-4R-CC and $-6 \mathrm{R}-\mathrm{CC}$ is characterized by an abundant occurrence of small unornamented Stilostomella spp. Other common species are Chilostomella sp., Pyrgo murrhina, Uvigerina hispida, Cassidulina sp. B, Pleurostomella sp., and Cibicidoides sp. This fauna indicates a depositional water depth of 2-3 km. The abundant occurrence of anaerobic fauna (Bernhard, 1986; Kaiho, 1989, in press) composed of small unornamented Stilostomella spp., Chilostomella sp., and Pleurostomella sp., and few occurrences of aerobic fauna in three samples from 126$788 \mathrm{C}-28 \mathrm{X}-\mathrm{CC}$ indicate a low- to medium-dissolved oxygen content. The fauna from other samples has a higher ratio of aerobic fauna than the fauna from Sample 126-788C-28X-CC and shows a medium oxygen content.

\section{Forearc Site 792}

Cenozoic strata at Site 792 are subdivided biostratigraphically into 12 zonules based on benthic foraminifers (Fig. 6).

\section{Rhabdammina sp. Assemblage Zonule}

Characteristic species: Agglutinated foraminifers such as Rhabdammina sp., Rhizammina sp., Reophax sp., Thalmannammina conglobata, and Haplophragmoides sp.; barren of calcareous foraminifers.

Abundance: Few.

Samples: $126-792 \mathrm{E}-64 \mathrm{R}-1,131-134 \mathrm{~cm},-64 \mathrm{R}-1,17-18 \mathrm{~cm}$, and $-63 R-C C$.

Age: early Oligocene.

Depositional water depth: Between 3.5 and $5 \mathrm{~km}$.

Dissolved oxygen: ?

\section{Barren Zonule}

Samples: From 126-792E-62R-CC through -59R-CC. Age: early Oligocene.

\section{Stilostomella spp.-Oridorsalis umbonatus Assemblage Zonule}

Characteristic species: Stilostomella spp., Oridorsalis umbonatus, and Pleurostomella spp.

Abundance: Few.

Samples: $126-792 \mathrm{E}-57 \mathrm{R}-\mathrm{CC}$ and $-57 \mathrm{R}-1,97-99 \mathrm{~cm}$.

Age: late Oligocene.

Depositional water depth: Between 2.5 and $3.5 \mathrm{~km}$.

Dissolved oxygen: High.

\section{Barren Zonule}

Samples: From 126-792E-56R-3, 25-27 cm, through -37R-CC. Age: late Oligocene. 


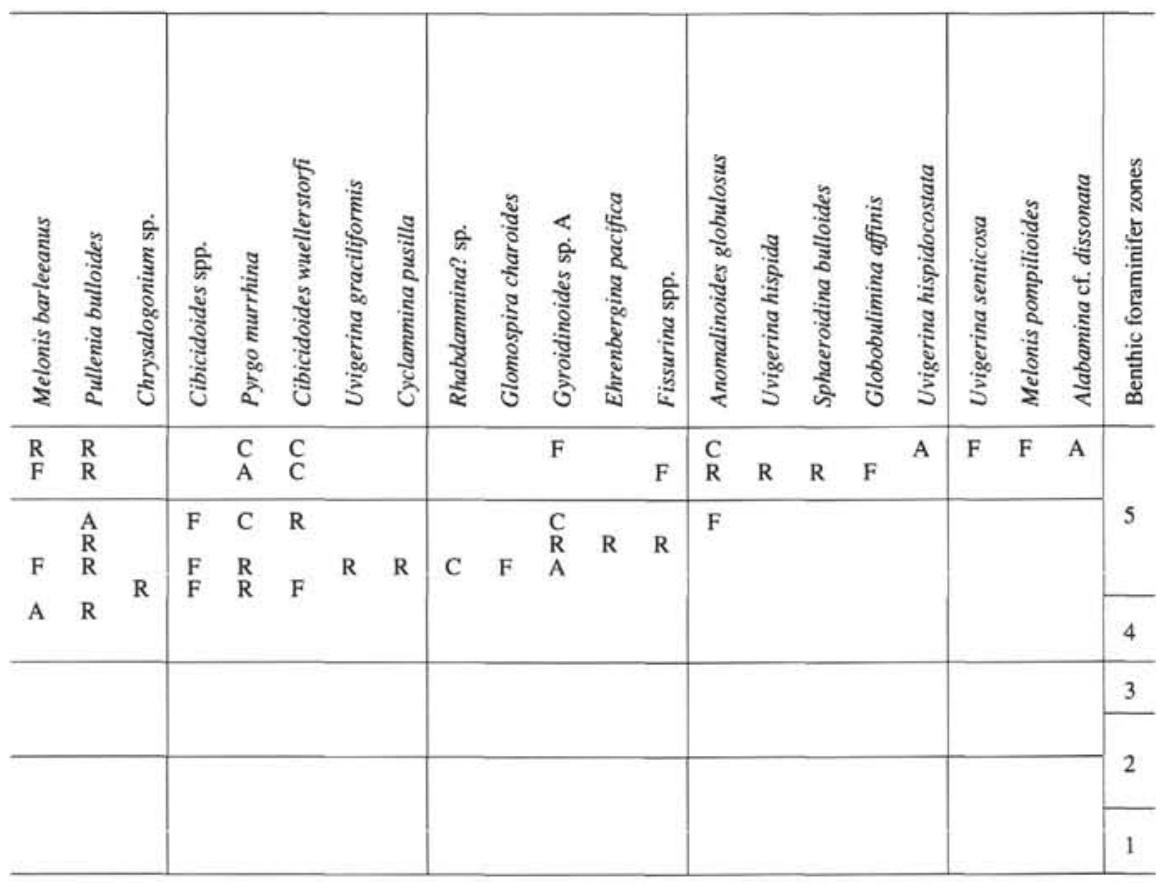

\section{Rhabdammina sp.-Stilostomella spp. Assemblage Zonule}

Characteristic species: Rhabdammina sp., Stilostomella spp., and Nodosaria longiscata.

Abundance: Few.

Samples: From 126-792E-36R-CC through -30R-CC.

Age: late Oligocene.

Depositional water depth: Between 2 and $3.5 \mathrm{~km}$.

Dissolved oxygen: ?

\section{Oridorsalis umbonatus-Pleurostomella spp. Assemblage Zonule}

Characteristic species: Oridorsalis umbonatus, Pleurostomella spp., Stilostomella spp., and Cibicidoides spp.

Abundance: Few.

Samples: From 126-792E-28R-CC through -24R-1, 61-63 cm.

Age: early Miocene.

Depositional water depth: Between 2 and $3.5 \mathrm{~km}$.

Dissolved oxygen: High.

\section{Cibicidoides renzi-Cibicidoides wuellerstorfi Assemblage Zonule}

Characteristic species: Cibicidoides renzi, Cibicidoides wuellerstorfi, Oridorsalis umbonatus, Pleurostomella spp. and Stilostomella spp.

Abundance: Few to common.

Samples: 126-792E-23R-1, 59-61 cm, and -22R-CC.

Age: middle Miocene.

Depositional water depth: Between 2 and $3.5 \mathrm{~km}$.

Dissolved oxygen: High.

\section{Cibicidoides spp.-Epistominella sp. Assemblage Zonule}

Characteristic species: Cibicidoides spp. and Epistominella sp. Abundance: Rare.

Samples: From 126-792E-22R-3, 60-65 cm, through $-17 \mathrm{R}-3$, $60-63 \mathrm{~cm}$

Age: middle Miocene.
Depositional water depth: Between 2 and $3.5 \mathrm{~km}$. Dissolved oxygen: High.

\section{Uvigerina graciliformis Assemblage Zonule}

Characteristic species: Uvigerina graciliformis, Cibicidoides spp., Sphaeroidina bulloides, Oridorsalis umbonatus, Gyroidinoides sp., Bulimina rostrata, Pleurostomella spp., Cibicidoides wuellerstorfi, Melonis barleeanus, and Lenticulina sp.

Abundance: Common.

Samples: 126-792E-16R-CC and -15R-CC.

Age: middle Miocene.

Depositional water depth: Between 2 and $3.5 \mathrm{~km}$.

Dissolved oxygen: High.

\section{Stilostomella spp.-Cibicidoides wuellerstorfi Assemblage Zonule}

Characteristic species: Stilostomella spp., Cibicidoides spp., and Cibicidoides wuellerstorfi.

Abundance: Few.

Samples: From 126-792E-15R-1, 115-117 cm, through -7R-CC.

Age: late Miocene.

Depositional water depth: Between 2 and $3.5 \mathrm{~km}$.

Dissolved oxygen: High.

\section{Stilostomella spp.-Pleurostomella spp. Assemblage Zonule}

Characteristic species: Stilostomella spp., Oridorsalis umbonatus, Cibicidoides wuellerstorfi, Pleurostomella spp., Sigmoilopsis schlumbergeri, Pullenia bulloides, Uvigerina hispidocostata, Uvigerina senticosa, Lenticulina spp., Amphicoryna scalaris (lower part of this zonule), and Bolivinita quadrilatera (middle part of this zonule).

Abundance: Abundant.

Samples: From 126-792E-6R-1, 58-60 cm, through 126-792A$8 \mathrm{H}-1,93-97 \mathrm{~cm}$.

Age: Pliocene to Pleistocene.

Depositional water depth: Between 2 and $3 \mathrm{~km}$.

Dissolved oxygen: High. 


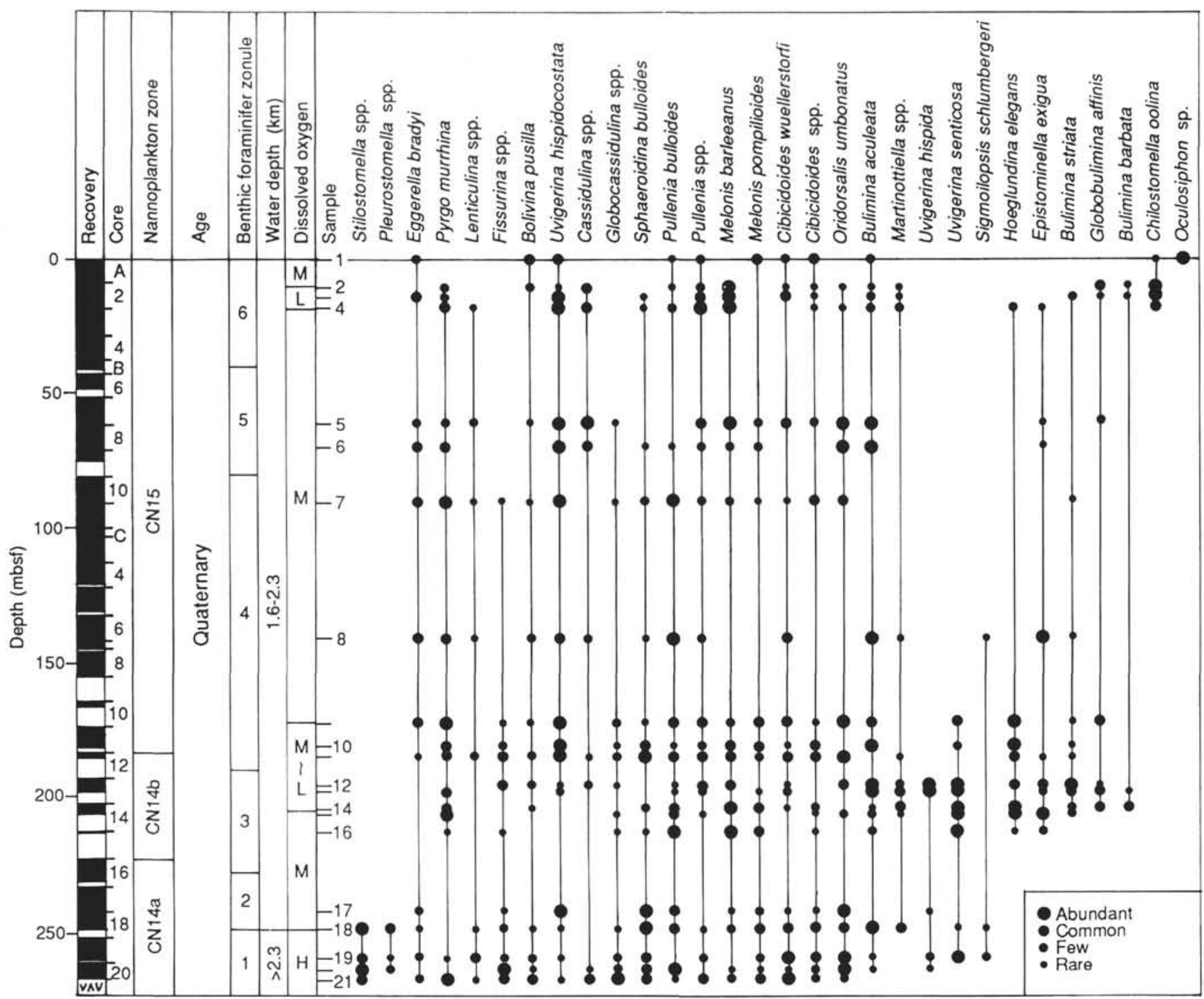

Figure 3. Stratigraphic distribution of benthic foraminifers, Site 790.

\section{Bulimina aculeata Assemblage Zonule}

Characteristic species: Melonis barleeanus, Pullenia bulloides, Bulimina aculeata, Uvigerina hispidocostata, Oridorsalis umbonatus, Sphaeroidina bulloides, Chilostomella oolina, Cibicidoides wuellerstorfi, Uvigerina hispida, and Globobulimina affinis.

Abundance: Abundant.

Samples: From 126-792A-7H-1, 59-61 cm, through -1H-CC.

Age: Pleistocene.

Depositional water depth: Between 1.6 and $2.3 \mathrm{~km}$.

Dissolved oxygen: High.

Remarks: The depositional age of the base of this assemblage zonule is approximately $0.7 \mathrm{Ma}$ based on magnetic datums. The same faunal change occurred at the same time at Sites 790 and 791.

\section{Forearc Site 793}

Cenozoic strata at Site 793 were subdivided biostratigraphically into seven zonules based on benthic foraminifers (Fig. 7), as follows.

\section{Rhabdammina spp.-Thalmannammina conglobata Assem- blage Zonule}

Characteristic species: Deep-water agglutinated foraminifers such as Rhabdammina spp., Thalmannammina conglobata, and Cyclammina trullissat a from mudstones, and reworked larger inner sublittoral benthic foraminifers from coarse sandstones.

Abundance: Few agglutinated foraminifers; larger foraminifers are rare in Cores 126-793B-72R to $-67 \mathrm{R}$ and common in Cores 126-793B-66R to -64R.

Samples: Samples 126-793B-74R-1, 5-7 cm, -73R-CC, and $-67 \mathrm{R}-1,2-4 \mathrm{~cm}$, from mudstones.

Age: late early Oligocene.

Depositional water depth: Between 3.5 and $5 \mathrm{~km}$.

Dissolved oxygen: ?.

Remarks: The present zonule is characterized by primitive agglutinated foraminifers and the absence of calcareous smaller benthic foraminifers. These benthic foraminifers show below CCD depositional water depths. Larger foraminifers from sandstones may be reworked from the inner neritic zone into the abyssal depth. 


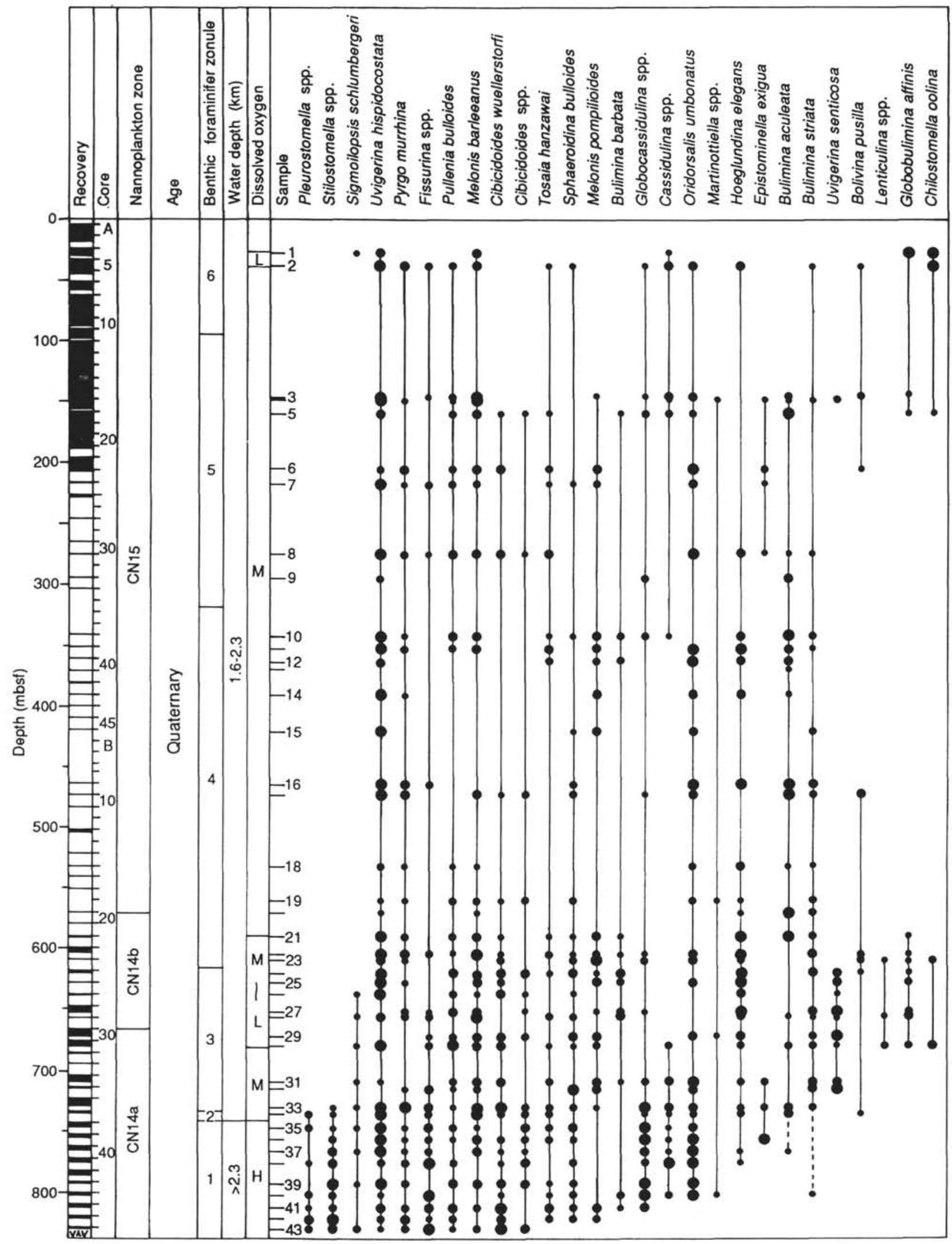

Figure 4. Stratigraphic distribution of benthic foraminifers, Site 791. 


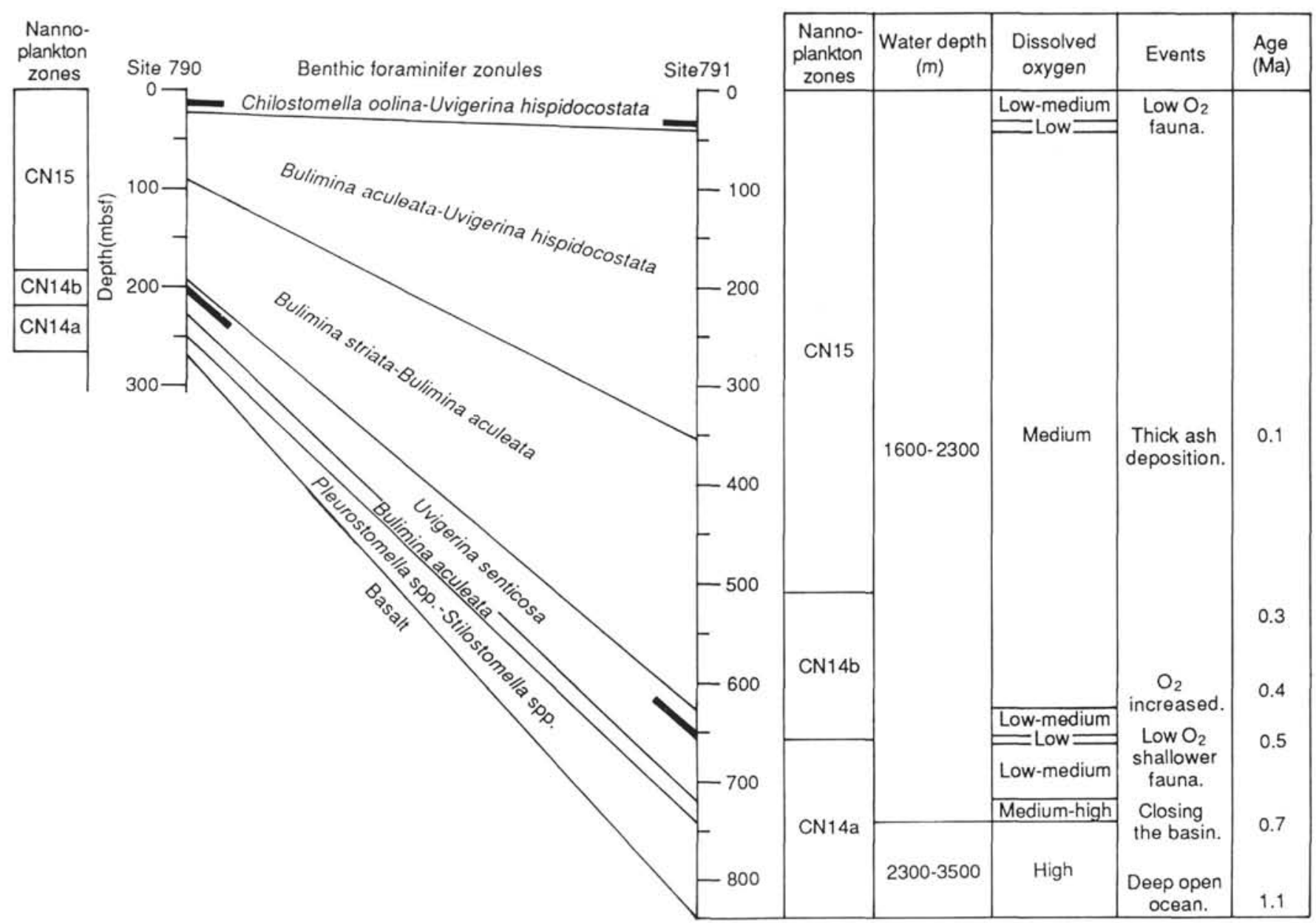

Figure 5. Quaternary benthic foraminifer zonules, paleowater depths, dissolved oxygen, and events in the Sumisu Rift. Black bars show low-oxygen horizons.

\section{Rhizammina sp.-Rhabdammina sp. Assemblage Zonule}

Characteristic species: Rhizammina sp., Rhabdammina sp., Thalmannammina conglobata, Cyclammina trullissata, and Hyperlammina sp. A.

Abundance: Few.

Samples: From 126-793B-61R-CC through -53R-CC.

Age: late early Oligocene-early late Oligocene.

Depositional water depth: Between 3.5 and $5 \mathrm{~km}$.

Dissolved oxygen: ?.

Remarks: This assemblage zonule is composed of primitive agglutinated foraminifers. This assemblage indicates a depositional water depth below the CCD.

\section{Stilostomella sp.-Hyperammina sp. A Assemblage Zonule}

Characteristic species: Rhizammina sp., Stilostomella sp., Hyperlammina sp. A, Glomospira gordialis, Reophanusoviculus (Brady), Rhabdammina sp., Labrospira sp., Trochammina sp., and Pleurostomella spp.

Abundance: Few.

Samples: From 126-793B-52R-2, 33-35 cm, through -36R-2, $108-111 \mathrm{~cm}$.

Age: early late Oligocene.

Depositional water depth: Between 3 and $3.5 \mathrm{~km}$.

Dissolved oxygen: ?.

Remarks: The assemblage, which consists of primitive agglutinated foraminifers plus Stilostomella sp. and Pleurostomella sp., shows above the CCD depositional water depths.

\section{Rhabdammina spp.-Oridorsalis umbonatus Assemblage Zonule}

Characteristic species: Rhabdammina spp., Rhizammina sp., Stilostomella spp., Thalmannammina conglobata, Gyroidinoides sp., Oridorsalis umbonatus, Pullenia spp., Reophax spp., and Glomospira gordialis.

Abundance: Few; common in Samples 126-793B-35R-CC and $-35 \mathrm{R}-3,109-111 \mathrm{~cm}$.

Samples: From 126-793B-35R-CC through -18R-3, 102-104 cm.

Age: late Oligocene.

Depositional water depth: Between 2 and $3.5 \mathrm{~km}$.

Dissolved oxygen: ?.

Remarks: The zonule is marked by the occurrence of primitive agglutinated foraminifers, Stilostomella spp., plus deep-sea trochospiral and planispiral calcareous forms such as Oridorsalis umbonatus, Gyroidinoides sp., and Pullenia spp.

\section{Cibicidoides spp.-Rhizammina sp. Assemblage Zonule}

Characteristic species: Stilostomella spp., Cibicidoides spp., Globocassidulina subglobosa, Rhizammina sp., Oridorsalis umbonatus, Pullenia bulloides, Gyroidinoides sp., Pullenia spp., Pleurostomella spp., Anomalinoides spp., Rhabdammina sp., Bulimina grata, and Eggerella sp.

Abundance: Abundant in Samples 126-793B-17R-4, 59-60 cm, and -16R-CC; few in Samples 126-793B-16R-1, 70-72 cm, and $-12 R-C C$.

Samples: From 126-793B-17R-4, 59-60 cm, through -12R-CC.

Age: early Miocene.

Depositional water depth: Between 2 and $3.5 \mathrm{~km}$. 


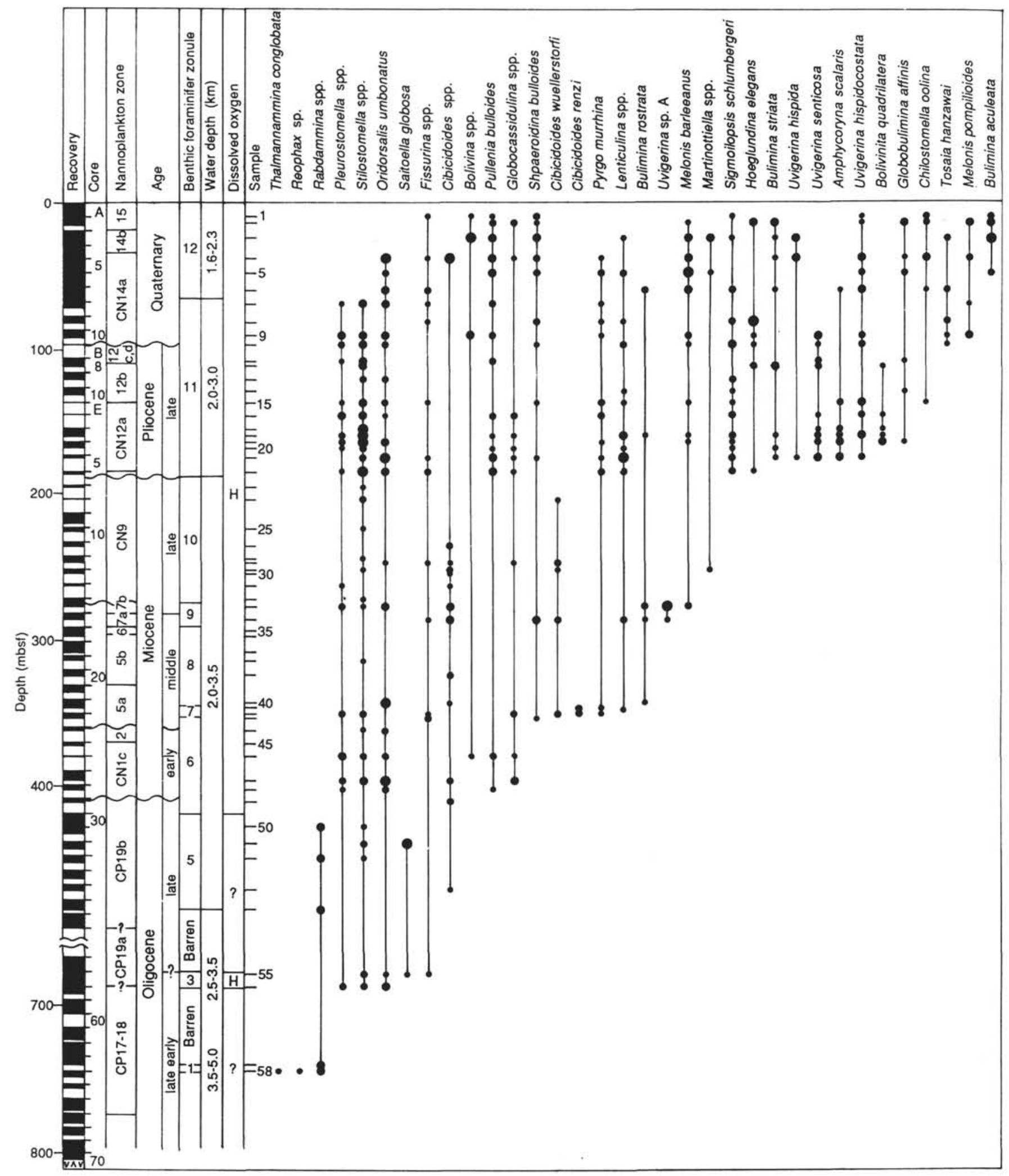

Figure 6. Stratigraphic distribution of benthic foraminifers, Site 792. 
Dissolved oxygen: High.

Remarks: This zonule is characterized by the continued occurrence of the fauna characterizing the subjacent zonule and the dominance of such calcareous foraminifers as Pullenia bulloides, Cibicidoides spp., Anomalinoides spp., and Globocassidulina subglobosa. Complex agglutinated foraminifers such as Vulvulina sp. and Eggerella sp. have their first occurrence in this zonule.

\section{Stilostomella spp.-Pullenia bulloides Assemblage Zonule}

Characteristic species: Stilostomella spp., Oridorsalis umbonatus, Pullenia bulloides, Globocassidulina subglobosa, Gyroidinoides spp., Anomalinoides spp., Cibicidoides spp., Pullenia spp. and Chrysalogonium longicostatum.

Abundance: Common below Sample 126-793B-8R-1, 60-62 cm; few above Sample 126-793B-7R-CC.

Samples: From 126-793B-12R-2, 46-49 cm, through -2R-1, 36$38 \mathrm{~cm}$.

Age: late early Miocene-early middle Miocene.

Depositional water depth: Between 2 and $3.5 \mathrm{~km}$.

Dissolved oxygen: High.

Remarks: This zonule is characterized by the fauna continued from the subjacent zonule but without such primitive agglutinated foraminifer genera as Rhizammina, Rhabdammina, and Bathysiphon.

\section{Pullenia bulloides-Melonis barleeanus Assemblage Zonule}

Characteristic species: Pullenia bulloides, Melonis barleeanus, Epistominella exigua, Melonis pompilioides, Oridorsalis umbonatus, Cibicidoides wuellerstorfi, Sphaeroidina bulloides, Pyrgo murrhina, Tosaia hanzawai, and Uvigerina senticosa.

Abundance: Abundant.

Samples: From 126-793A-11H-CC through -3H-4, 0-2 cm.

Age: Pleistocene.

Depositional water depth: Between 2.3 and $3.5 \mathrm{~km}$.

\section{Outer-arc High Sites $\mathbf{7 8 2}$ and 786}

At Sites 782 and 786 drilled in the outer-arc high, benthic foraminifers occurred abundantly in the upper Eocene through Holocene sequences and their preservation is quite good. Faunal compositions and the pattern of benthic foraminifer changes are similar between these two sites (Table 3). Cenozoic strata recovered from these sites are subdivided biostratigraphically into five zonules based on benthic foraminifers.

\section{Nuttallides truempyi-Osangularia mexicana Assemblage Zonule}

Characteristic species: Nuttallides truempyi (Nuttall), Osangularia mexicana (Cole), Bulimina jarvisi Cushman and Parker, Oridorsalis umbonatus, Stilostomella spp., and Pleurostomella spp.

Abundance: Abundant.

Samples: $125-786 \mathrm{~A}-11 \mathrm{X}-3,50-58 \mathrm{~cm}$, and $125-782 \mathrm{~A}-41 \mathrm{X}-3$, $52-60 \mathrm{~cm}$.

Age: late Eocene.

Depositional water depth: Between 1.3 and $2.1 \mathrm{~km}$. Tjalsma and Lohmann's (1983) data show that $O$. mexicana was distributed abundantly between 1.3 and $2.1 \mathrm{~km}$ water depths at P15 Zone time. The other species support this water-depth range.

Dissolved oxygen: High.

\section{Osangularia mexicana-Globocassidulina subglobosa Assem- blage Zonule}

Characteristic species: Oridorsalis umbonatus, Osangularia mexicana, Stilostomella spp., Globocassidulina subglobosa, Gyroidinoides sp. A, and Pleurostomella spp.
Abundance: Abundant.

Samples: $125-782 \mathrm{~A}-40 \mathrm{X}-2,47-55 \mathrm{~cm}, 125-786 \mathrm{~A}-10 \mathrm{X}-6,32-$ $40 \mathrm{~cm}$, and $125-782 \mathrm{~A}-39 \mathrm{X}-2,47-55 \mathrm{~cm}$.

Age: latest Eocene-early Oligocene.

Depositional water depth: Between 1.3 and $2.1 \mathrm{~km}$.

Dissolved oxygen: High.

\section{Cibicidoides renzi-Bulimina jarvisi Assemblage Zonule}

Characteristic species: Oridorsalis umbonatus, Stilostomella spp., Cibicidoides renzi, Bulimina jarvisi, Pleurostomella spp., Gyroidinoides sp. A, Osangularia mexicana, and Globocassidulina subglobosa.

Abundance: Abundant.

Samples: $125-782 \mathrm{~A}-37 \mathrm{X}-2,47-55 \mathrm{~cm}$, and 125-786A-9X-5, 100$108 \mathrm{~cm}$.

Age: late Oligocene.

Depositional water depth: Between 1.3 and $3.0 \mathrm{~km}$.

Dissolved oxygen: High.

\section{Oridorsalis umbonatus-Stilostomella spp. Assemblage Zonule}

Characteristic species: Oridorsalis umbonatus, Stilostomella spp., Pleurostomella spp., Globocassidulina subglobosa, and Melonis barleeanus.

Abundance: Abundant.

Samples: $125-782 \mathrm{~A}-35 \mathrm{X}-2,52-60 \mathrm{~cm}$, and $125-786 \mathrm{~A}-8 \mathrm{X}-1,51-$ $55 \mathrm{~cm}$.

Age: early middle Miocene.

Depositional water depth: Between 1.3 and $3.0 \mathrm{~km}$.

Dissolved oxygen: High.

\section{Cibicidoides wuellerstorfi-Pyrgo murrhina Assemblage Zonule}

Characteristic species: Oridorsalis umbonatus, Pyrgo murrhina, Cibicidoides wuellerstorfi, Stilostomella spp. (Miocene), Gyroidinoides sp. A, Anomalinoides globulosus (late Miocene-Pleistocene), and Pullenia bulloides.

Abundance: Abundant.

Samples: From 125-782A-31X-2, 87-95 cm, through -2H-2, 120$122 \mathrm{~cm}$, and $125-786 \mathrm{~A}-7 \mathrm{X}-2,100-108 \mathrm{~cm}$, and $-6 \mathrm{X}-2,61-69 \mathrm{~cm}$.

Age: middle middle Miocene-Pleistocene.

Depositional water depth: Between 2.0 and $3.5 \mathrm{~km}$.

Dissolved oxygen: High.

\section{DISCUSSION}

\section{Sumisu Rift}

Deep open-ocean benthic foraminifer fauna $(>2300 \mathrm{~m})$ inhabited this area between 1.0 and $0.7 \mathrm{Ma}$. The appearance of the low- to mediumoxygen shallower fauna $(1600-2300 \mathrm{~m})$ from 0.7 to 0.5 Ma suggests that a closure of the basin may have started at this time as a result of the development of volcanoes and rift flank uplifts around the basin. The slight change of the fauna at $0.4 \mathrm{Ma}$ suggests that dissolved oxygen slightly increased at that time. After the deposition of thick volcanic ash layers at about $0.03 \mathrm{Ma}$, low-oxygen fauna occupied the basin.

\section{Rift Margin}

Benthic foraminifers indicate a depositional water depth between 2 and $3 \mathrm{~km}$ for Holes $788 \mathrm{C}$ and 788D in the early Pliocene. These data indicate about $0.6-1.6 \mathrm{~km}$ of total basement uplift. The uplift may have led to the formation of the major hiatus between 2.3 and $0.3 \mathrm{Ma}$ (Fig. 8). Most assemblages from these holes suggest a low to medium dissolved-oxygen content. 


\section{Forearc Basin}

Benthic foraminifer faunal changes at Site 792 in the forearc basin show a shallowing water depth from below the CCD (about $3.5 \mathrm{~km}$ ) in the late early Oligocene to the present depth of $1800 \mathrm{~m}$. These data suggest about $1 \mathrm{~km}$ of total basement uplift at Site 792 in the inner part of the forearc basin since about $31 \mathrm{Ma}$ (Fig. 9).

At Site 793, the benthic foraminifer faunal changes show shallowing water depths from about $4 \mathrm{~km}$ in the early Oligocene to $2.3-3.5 \mathrm{~km}$ in the present. As the present water depth is $3 \mathrm{~km}$, this estimate means about $0.8-\mathrm{km}$ shallowing of the seafloor and about $0.6 \mathrm{~km}$ of the total basement subsidence occurred since about $30 \mathrm{Ma}$ (Fig. 10).

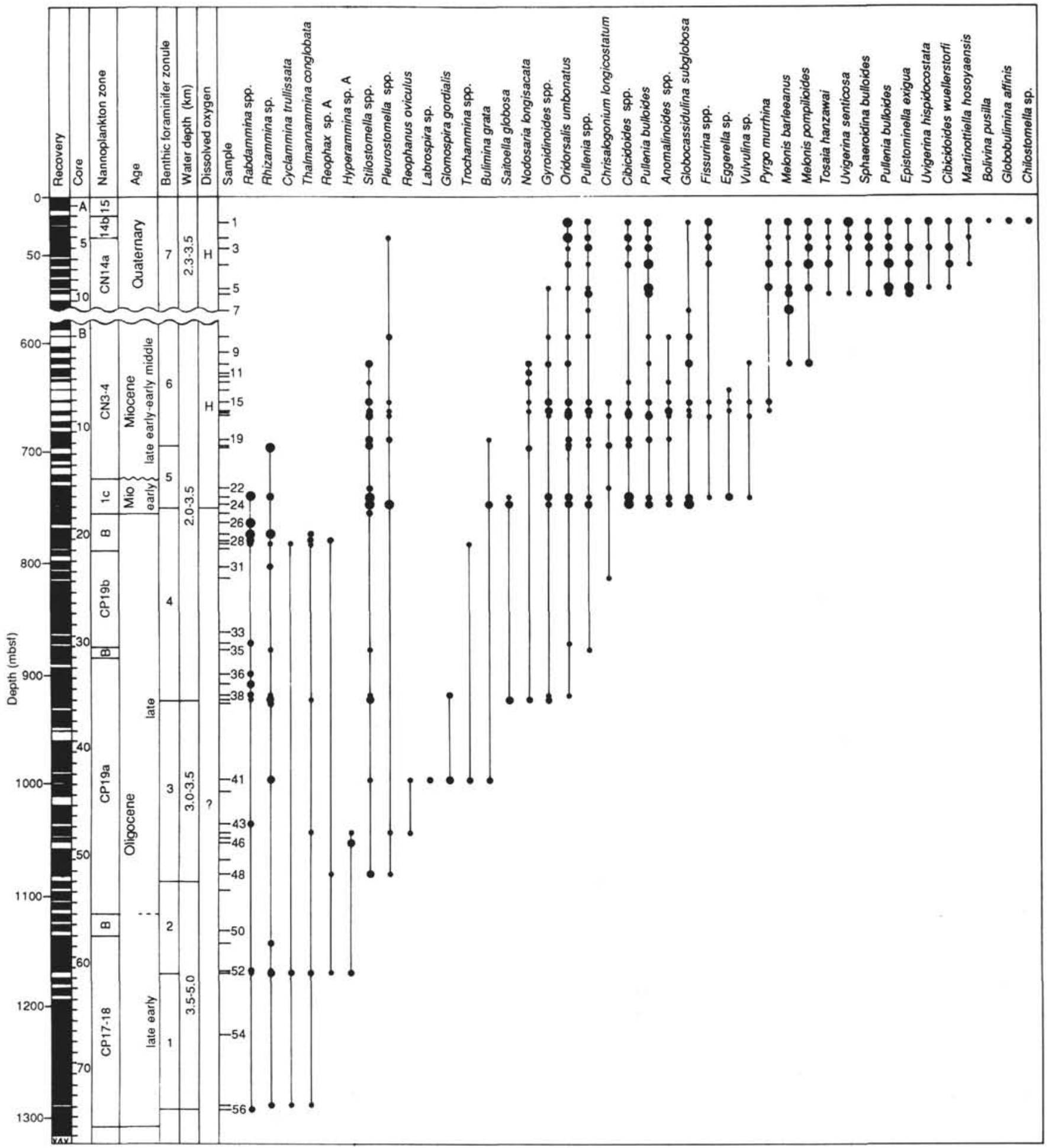

Figure 7. Stratigraphic distribution of benthic foraminifers, Site 793. 
The former uplift led to a thinner sediment accumulation $(800 \mathrm{~m})$ and the latter subsidence to a thicker sediment accumulation ( $1400 \mathrm{~m})$ at these sites.

Early Oligocene larger foraminifer faunas comprising such species as Nummulites vascus Joly and Leymerie and Eulepidina formosa (Schlumberger) (K. Matsumaru, pers. comm., 1990) were found in layers of sandstones recovered from depths between 1200 and $1300 \mathrm{~m}$ below the seafloor of the Izu-Bonin forearc region at Site 793. These faunas are composed of nine larger and two smaller foraminifer species that inhabited the inner sublittoral depth zone. Siltstones intercalated with the sandstone contain late early Oligocene nannofossils (CP17-CP18) as well as such abyssal benthic foraminifer genera as Rhabdammina and Rhizammina. These data are taken to indicate that the larger foraminifer faunas were transported from the inner sublittoral zone to the abyssal zone in late early Oligocene time.

\section{Outer-arc High}

At Sites 782 and 786 drilled in the outer-arc high, benthic foraminifers indicate a deepening water depth from 1.3 to $2.1 \mathrm{~km}$ in the late Eocene to the present depth of about $3 \mathrm{~km}$. These data suggest about 1.1-1.9 and $1.3-2.1 \mathrm{~km}$ of total basement subsidence at Sites 786 and 782, respectively (Fig. 11).

\begin{abstract}
All Areas
Benthic foraminifer faunas in all the zonules below the first occurrence (not first evolutionary appearance) of Bulimina aculeata that contain Pleurostomella and Stilostomella indicate a deep-ocean basin environment (see Woodruff, 1985, table 5). However, the fauna containing $B$. aculeata shows a marginal ocean environment based on many data (e.g., Kaiho and Hasegawa, 1986; Akimoto, 1989; Yasuda, 1989). The FO of B. aculeata and the LO of Stilostomella spp. and Pleurostomella spp. consistently occurred at $0.7 \mathrm{Ma}$ at all three sites $(790,791$, and 792$)$. This fact is taken to suggest a change of water mass from the one originating from the central part of the ocean to that originating from ocean margin areas at this time.
\end{abstract}

\section{CONCLUSIONS}

The benthic foraminifer fauna at two Sumisu Rift sites indicates that a deep open-ocean $(>2300 \mathrm{~m})$ or a basin with open-ocean access existed between 1.1 and $0.7 \mathrm{Ma}$ at the time of initiation of the rifting. A low- to medium-oxygen fauna (1600-2300 m) appeared from 0.7 to $0.5 \mathrm{Ma}$. This fact suggests that a basin without open-ocean access may have originated at this time.

Lower bathyal benthic faunas from lower Pliocene sediments of the rift margin site show about $0.6-1.6 \mathrm{~km}$ of total basement uplift. This uplift may have led to the formation of the major hiatus between 2.3 and $0.3 \mathrm{Ma}$.

Benthic foraminifer faunal changes at two sites in the forearc basin show a shallowing water depth from below the CCD (about $3.5 \mathrm{~km}$ ) in the late early Oligocene to the present depth of 1800 and $2975 \mathrm{~m}$, respectively. These data suggest about $1 \mathrm{~km}$ of total basement uplift in the inner part of the forearc basin and about $0.6 \mathrm{~km}$ total basement subsidence in the central part of the forearc basin since about $31 \mathrm{Ma}$. The former uplift led to a thinner sediment accumulation $(800 \mathrm{~m})$ and the latter subsidence a thicker sediment accumulation $(1400 \mathrm{~m})$.

Faunal changes of benthic foraminifers observed in two sites sequences drilled at the outer-arc high document a deepening water depth from 1.3 to $2.1 \mathrm{~km}$ in the late Eocene to the present depth of about $3 \mathrm{~km}$. These data indicate about $1-2 \mathrm{~km}$ of total basement subsidence in the outer-arc high since the late Eocene.

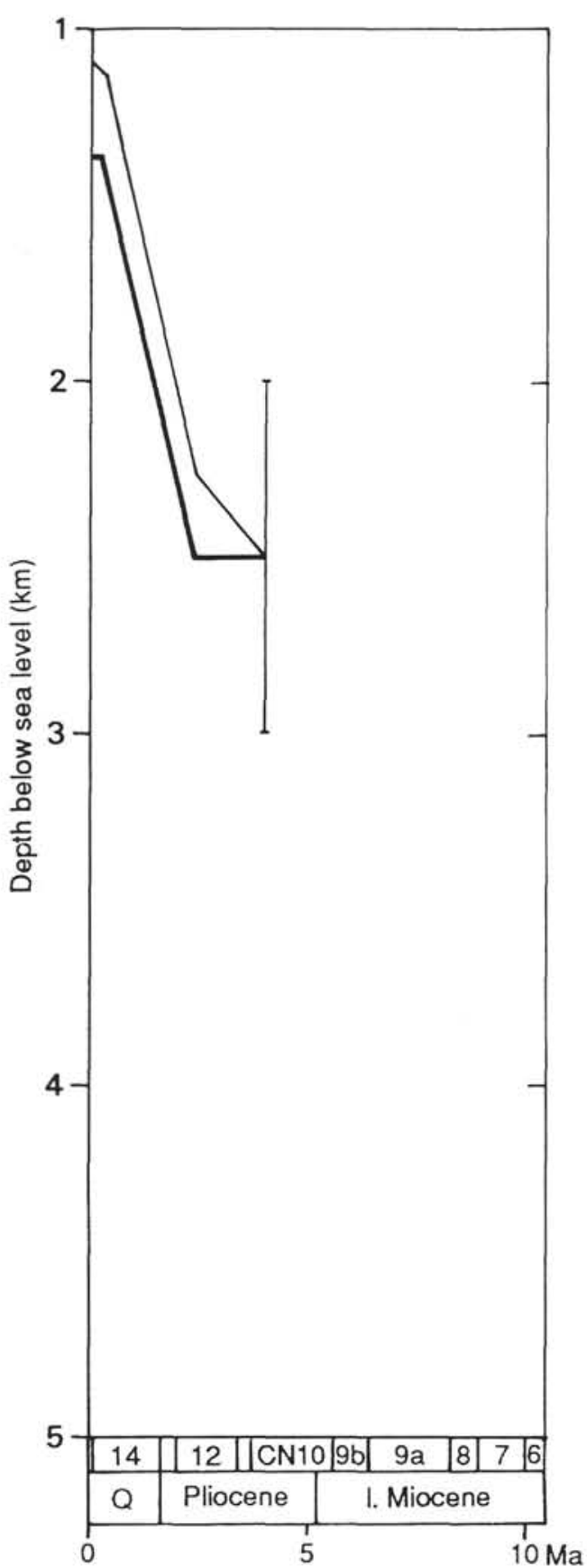

Figure 8. Paleobathymetry and motion of basement (heavy line), Site 788. These lines are based on a hypothesis that all uplift of basement occurred in hiatus periods. A vertical bar shows paleowater depths based on benthic foraminifer faunas.

These results are supportive of the total basement uplift in the inner part of the Bonin arc-trench system since the late Oligocene and the total basement subsidence in the outer part of the system since the late Eocene.

The LO of Stilostomella spp. and Pleurostomella spp. and the FO of Bulimina aculeata consistently occur at $0.7 \mathrm{Ma}$ at all sites between 1800 and $2300 \mathrm{~m}$ water depth. This fact is taken to suggest a change of water mass from the one originating from the central part of the ocean to that originating from ocean margin areas at this time. 


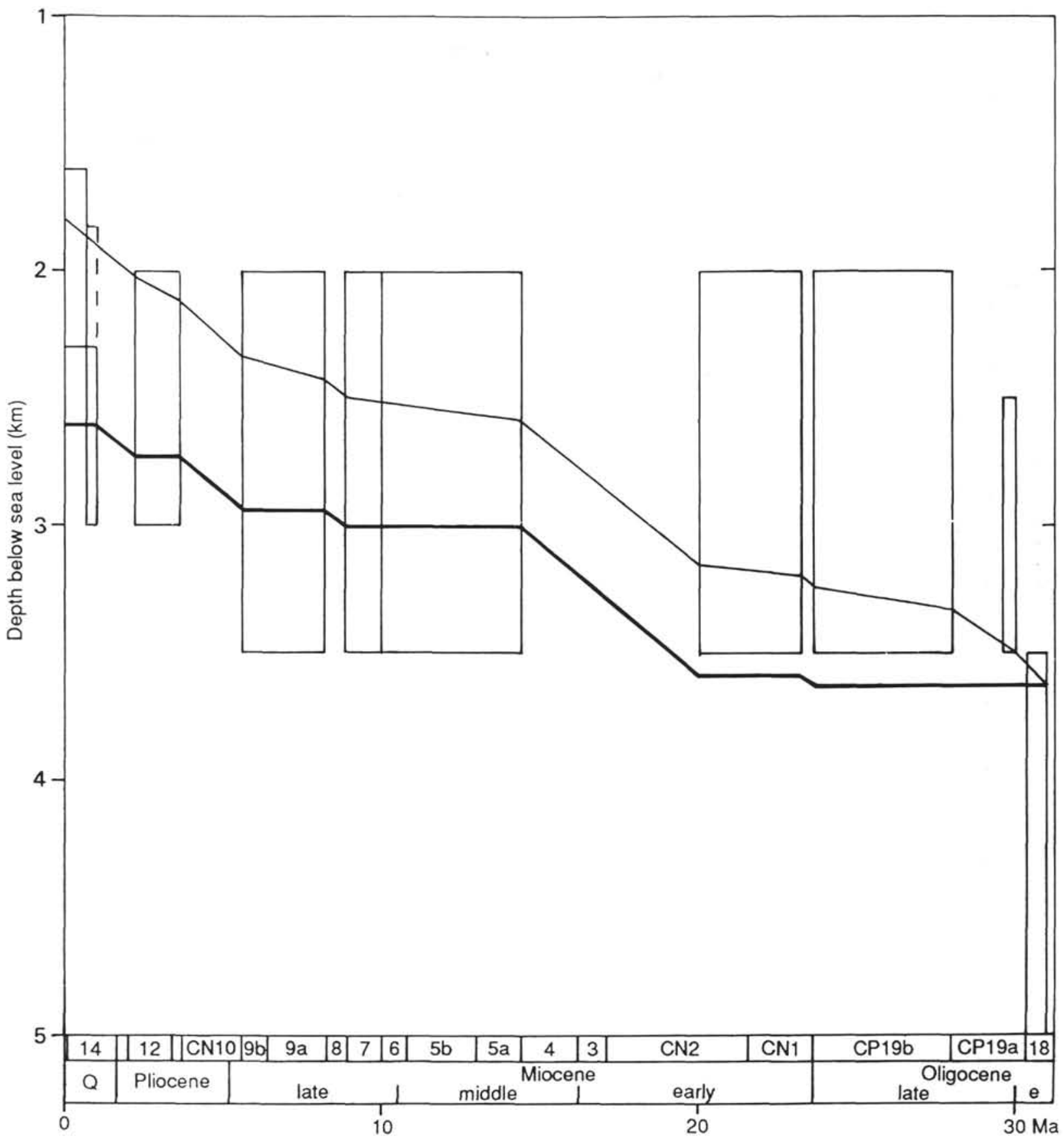

Figure 9. Paleobathymetry and motion of basement (heavy line), Site 792. These lines are based on a hypothesis that all uplift of basement occurred in hiatus periods. Squares show paleowater depths based on benthic foraminifer faunas. A square drawn by dashed lines shows possibility of lateral change of water mass.

\section{SYSTEMATIC DESCRIPTION OF NEW GENUS AND SPECIES}

Family Gavelinellidae Hofker, 1956

Subfamily Gyroidinoidinae Saidova, 1981

Genus Saitoella Kaiho, gen. nov.

Description. Test subglobular, chambers in a trochospirally arranged closed coil, only the final whorl visible on both spiral and umbilical sides; periphery rounded; sutures radial; wall calcareous, finely perforate, surface smooth; aperture an elongate interiomarginal slit, extending from near the periphery to the open umbilicus, bordered with a narrow lip.

Type species. Saitoella globosa Kaiho, gen. nov. et sp. nov.

Remarks. This genus is probably related to such genera as Rotaliatina Cushman (late Eocene-Oligocene) and Rotaliatinopsis Banner (PliocenePleistocene) but is distinguished in having an involute spiral side. The generic name is given in honor of Professor Tsunemasa Saito of Tohoku University, Japan, in recognition of his work on foraminifers. 


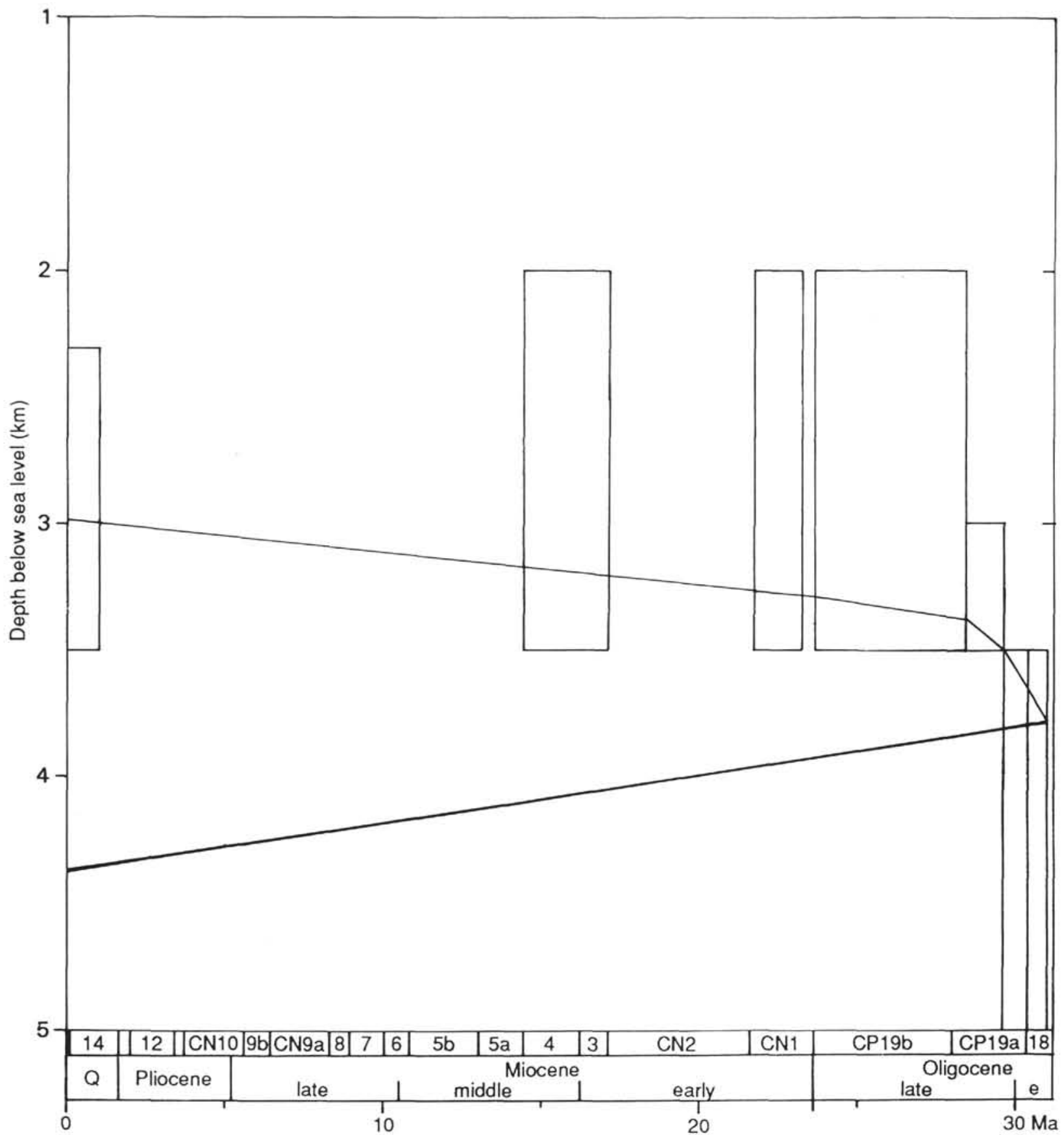

Figure 10. Paleobathymetry and motion of basement (heavy line), Site 793. Squares show paleowater depths based on benthic foraminifer faunas.

Saitoella globosa Kaiho, gen. nov. et sp. nov.

(Plate 7, Figs. 9a-c)

Description. Test subglobular, chambers in a trochospiral coil, but involute on both spiral and umbilical sides; periphery broadly rounded; chambers four in the final whorl, slightly lobulate; sutures radial, nearly straight, slightly depressed; wall calcareous, finely perforate, surface smooth with more and slightly larger pores on spiral side; aperture an elongate interiomarginal slit, extending from near the periphery to the deep open umbilicus, bordered with a narrow lip.

Occurrences. Saitoella globosa occurs in late Oligocene to early Miocene sediments from Sites 792 and 793.

Size. 0.3-0.4 mm, maximum diameter; holotype, $0.39 \mathrm{~mm}$.

Holotype. IGPS 101180 (Plate 7, Figs. 9a-c)

Paratypes. IGPS 101181 through 101186
Type locality. Forearc basin in the Izu-Bonin Arc, Northwest Pacific Ocean, Sample 126-792E-32R-1, 13-15 cm.

\section{Subfamily Gavelinellinae Hofker, 1956 \\ Genus Globogyroidina Kaiho, gen. nov.}

Description. Test trochospiral, biconvex, lenticular with globular final chamber; periphery broadly rounded on final chamber, subacute on the other part; sutures radial, flush with surface on both sides; wall calcareous, thin, finely perforate, surface smooth; aperture of final chamber a low slit, opens on both spiral and umbilical sides, individually bordered with a narrow flap; aperture except for one of final chamber a low interiomarginal slit, extending from near the periphery to about halfway toward the umbilicus, bordered with a narrow lip. 


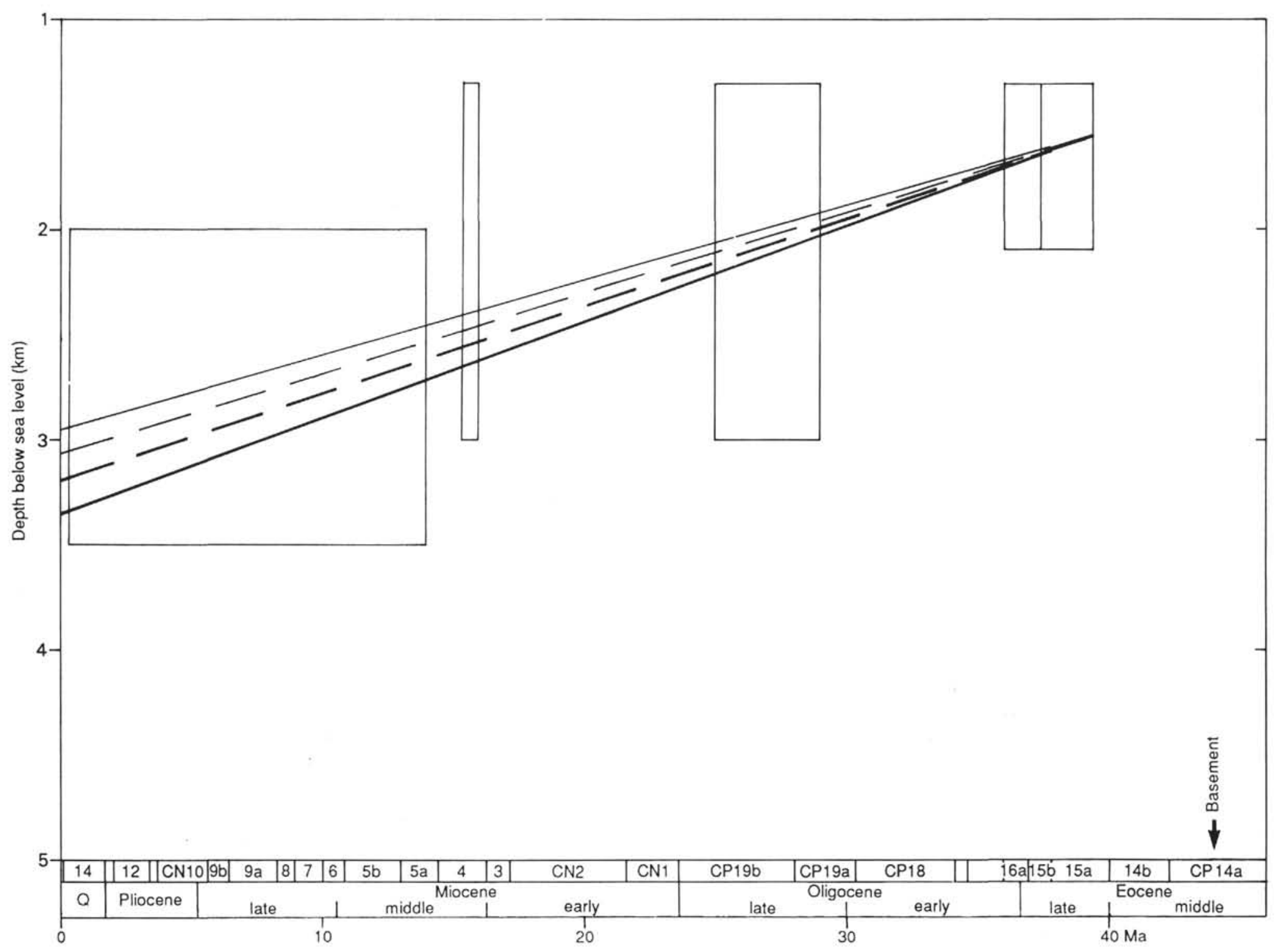

Figure 11. Paleobathymetry and motion of basement (heavy line), Sites 782 and 786. Squares show paleowater depths based on benthic foraminifer faunas. Dashed lines: Site 786. 
Type species. Globogyroidina boninensis Kaiho, gen. nov. et sp. nov. Remarks. This genus is similar to genus Gyroidina d'Orbigny (Holocene) but differs in having a large globular final chamber with a slitlike aperture developed separately on both spiral and umbilical sides and in having a finer and thinner wall.

Globogyroidina boninensis Kaiho, gen. nov. et sp. nov. (Plate 8, Figs. 9a-c)

Description. Test trochospiral, biconvex, lenticular with large globular final chamber; periphery broadly rounded on final chamber, subacute on the other part; chambers eight to ten in final whorl except for final chamber, slightly lobulate; final chamber strongly inflated, covering one-third of test; sutures radial, flush with surface on both sides, straight on umbilical side, oblique on spiral side; wall calcareous, thin, finely perforate, surface smooth; wall of final chamber very thin, transparent; aperture of final chamber a low slit, opened separately on both spiral and umbilical sides, bordered with a narrow flap; aperture except for one of final chamber a low interiomarginal slit, extending from near the periphery to about halfway toward the umbilicus, bordered with a narrow lip.

Occurrences. Globogyroidina boninensis occurs in a single sample of Quaternary sediments from Site 790.

Size. 0.5-0.6 mm, maximum diameter; holotype, $0.56 \mathrm{~mm}$.

Holotype. IGPS 101187 (Plate 8, Figs. 9a-c)

Paratypes. IGPS 101188 through 101191.

Type locality. Sumisu Rift, Northwest Pacific Ocean, Sample 126-790A$2 \mathrm{H}-3,98-100 \mathrm{~cm}$.

\section{ACKNOWLEDGMENTS}

I would like to thank the crew and the rest of the shipboard technical and scientific parties, especially the co-chief scientists, B. Taylor and K. Fujioka. I am very grateful to T. Saito, Y. Takayanagi, and B. Taylor for reviewing the manuscript. I am thankful to K. Matsumaru for identification of larger foraminifers and to S. Otomo for his photographic assistance.

\section{REFERENCES}

Akimoto, K., 1989. Recent foraminifera from Enshunada. In Takayanagi, Y., and Ishizaki, K. (Eds.), Collected Papers on Foraminifera from the Japanese Islands: Sendai (Toko Press), 93-104. (in Japanese)

Berggren, W. A., Kent, D. V., Flynn, J. J., and Van Couvering, J. A., 1985. Cenozoic geochronology. Geol. Soc. Am. Bull., 96:1407-1418.

Bernhard, J. M., 1986. Characteristic assemblages and morphologies of benthic foraminifera from anoxic, organic-rich deposits: Jurassic through Holocene. J. Foraminiferal Res., 16:207-215.

Boltovskoy, E., 1980. Benthic foraminifera of the bathyal zone from Oligocene through Quaternary. Rev. Esp. Micropaleontol., 12:283-304.

Egeberg, P. K., and Leg 126 Shipboard Scientific Party, 1990. Unusual composition of pore waters found in the Izu-Bonin forearc sedimentary basin. Nature, 344:215-218.
Fryer, P., Pearce, J. A., Stokking, L. B., et al., 1990. Proc. ODP, Init. Repts., 125: College Station, TX (Ocean Drilling Program).

Fujioka, K., Taylor, B., Nishimura, A., Koyama, M., Kaiho, K., Tazaki, K., Janecek, T., and Scientific Party of ODP Leg 126, 1989. Drilling across the Bonin Arc-results of ODP Leg 126 Cruise. Geogr. J. Jpn., 98:54-78. (In Japanese with English abstract)

Hofker, J., Sr., 1956. Die Globotruncanen von Nordwest-Deutschland und Holland. Neues Jahrb. Geol. Paleontol. Abh., 103:312-340.

Ingle, J. C., Jr., 1980. Cenozoic paleobathymetry and depositional history of selected sequences within the southern California continental borderland. Spec. Publ. Cushman Found. Foraminiferal Res., 19:163-195.

Kaiho, K., 1989. Morphotype changes of deep-sea benthic foraminifera during the Cenozoic Era and their paleoenvironmental implications. Kaseki, 47:123. (in Japanese with English abstract).

, 1991. Global changes of Paleogene aerobic/anaerobic benthic foraminifera and deep-sea circulation. Palaeogeogr., Palaeoclimatol., Palaeoecol., 83:65-85.

Kaiho, K. and Hasegawa, S., 1986. Bathymetric distribution of benthic foraminifera in bottom sediments off Onahama, Fukushima Prefecture, northeast Japan. In Matoba, Y., and Kato, M. (Eds.), Studies on Cenozoic Benthic Foraminifera: Akita (Akita University), 43-52. (In Japanese)

Leg 126 Shipboard Scientific Party, 1989. Arc volcanism and rifting. Nature, 342:18-20.

Murakami, F., 1988. Structural framework of the Sumisu Rift, Izu-Ogasawara Arc. Bull. Geol. Surv. Jpn., 39:1-21.

Saidova, K. M., 1981. On an up-to-date system of supraspecific taxonomy of Cenozoic benthonic foraminifera. Inst. Okeanol. P. P. Shirshova, Akad. Nauk SSSR.

Taylor, B., Fujioka, K., et al., 1990. Proc. ODP, Init. Repts., 126: College Station, TX (Ocean Drilling Program).

Tjalsma, L., and Lohmann, G. P., 1983. Paleocene-Eocene bathyal and abyssal benthic foraminifera from the Atlantic Ocean. Micropaleontol. Spec. Publ., 4:1-90.

van Andel, T. H., Heath, G. R., and Moore, T. C., Jr., 1975. Cenozoic history and paleoceanography of the central equatorial Pacific. Mem. Geol. Soc. Am., 143:1-134.

van Morkhoven, FP.C.M., Berggren, W. A., and Edwards, A. S., et al., 1986. Cenozoic cosmopolitan deep-water benthic foraminifera. Bull. Cent. Rech. Explor.-Prod. Elf-Aquitaine, Mem. 11.

Woodruff, F., 1985. Changes in Miocene deep-sea benthic foraminifera distribution in the Pacific Ocean: relationship to paleoceanography. In Kennett, J. P. (Ed.), The Miocene Ocean: Paleoceanography and Biogeography. Mem. Geol. Soc. Am., 163:131-175.

Yasuda, H., 1989. Benthic foraminifera and sedimentary facies of the lower slope basin off Shikoku, Japan. In Takayanagi, Y., and Ishizaki, K. (Eds), Collected Papers on Foraminifera from the Japanese Islands: Sendai (Toko Press), 83-92. (in Japanese)

Date of initial receipt: 1 November 1990

Date of acceptance: 24 June 1991

Ms 126B-137 


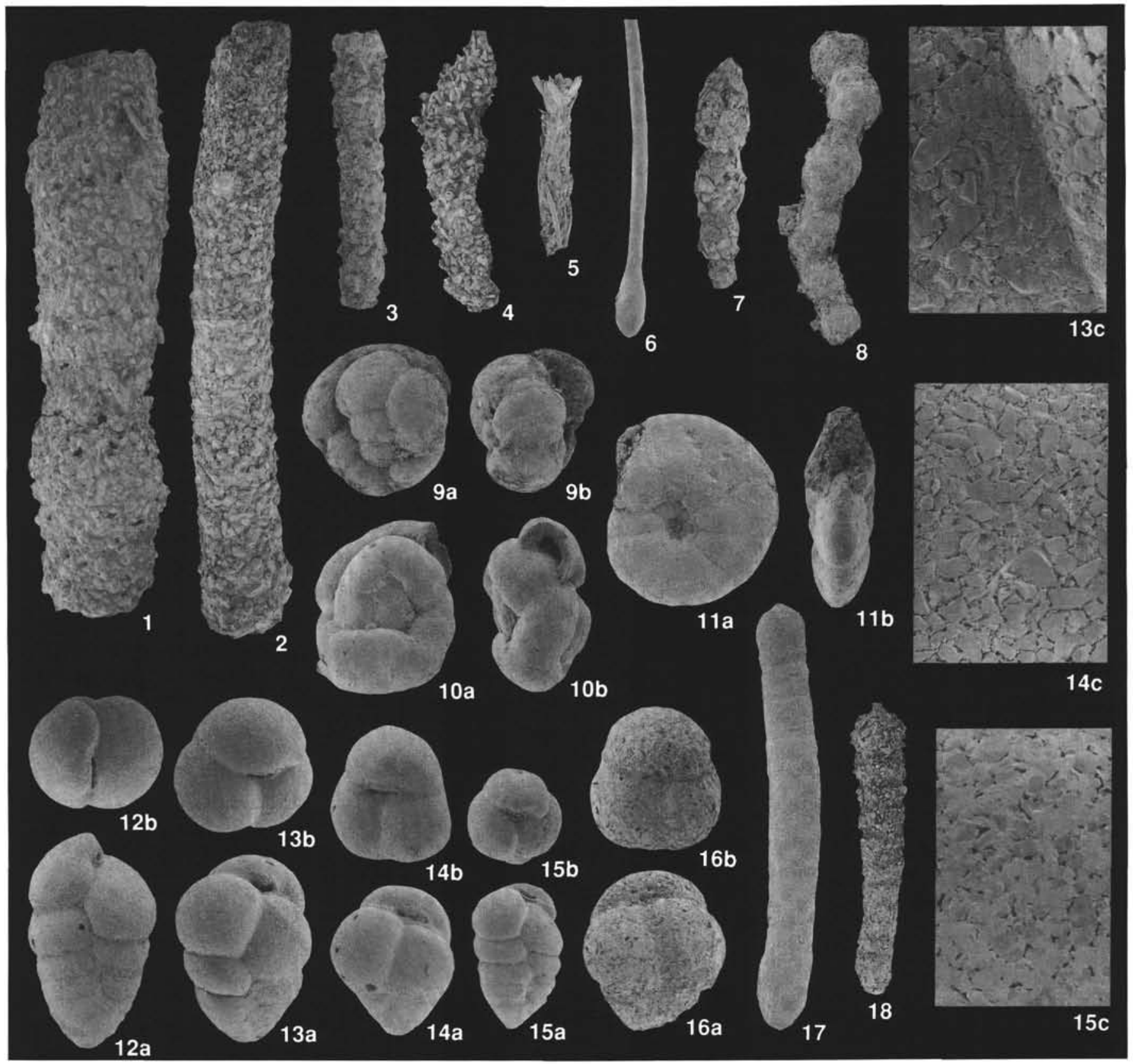

Plate 1. 1-2. Rhabdammina sp. A; (1) Sample 126-793B-16R-CC (23x); (2) Sample 126-793B-47R-CC (23x). 3. Rhabdammina sp. B; Sample 126-792E-64R1, 131-134 cm (23x). 4. Rhizammina sp.; Sample 126-792E-33R-1, 136-140 cm (23x). 5. Dendronina arborescens Heron-Allen and Earland; Sample 126-793B-61R-CC (23x). 6. Hyperammina sp. A; Sample 126-793B-61R-CC (36x). 7. Reophax sp. A; Sample 126-792E-30R-CC (23x). 8. Reophax aduncus Brady; Sample 126-792E-64R-1, 131-134 cm (23x). 9-10. Thalmannammina conglobata (Brady); (9) Sample 126-792E-64R-1, 131-134 cm (23x); (10) Sample 126-793B-21R-1, 1-2 cm (36×). 11. Cyclammina trullissata (Brady); Sample 126-793B-61R-CC (51×). 12. Karreriella bradyi (Cushman); Sample 126-790C17X-CC (36x). 13-15. Eggerella bradyi (Cushman); (13) Sample 126-790C-10X-CC (13a-b, 36x 13c, 513x); (14) Sample 126-790B-10H-CC (14a-b, 36x 14c, 513x); (15) Sample 126-790A-2H-3, 98-100 cm (15a-b, 36x 15c, 513×). 16. Eggerella propinqua (Brady); Sample 126-793B-16R-CC (36x). 17. Martinottiella communis (d'Orbigny); Sample 126-790B-3H-1, 0-1 cm (23×). 18. Martinottiella hosoyaensis Asano; Sample 126-790A-2H-3, 98-100 cm (23x). 


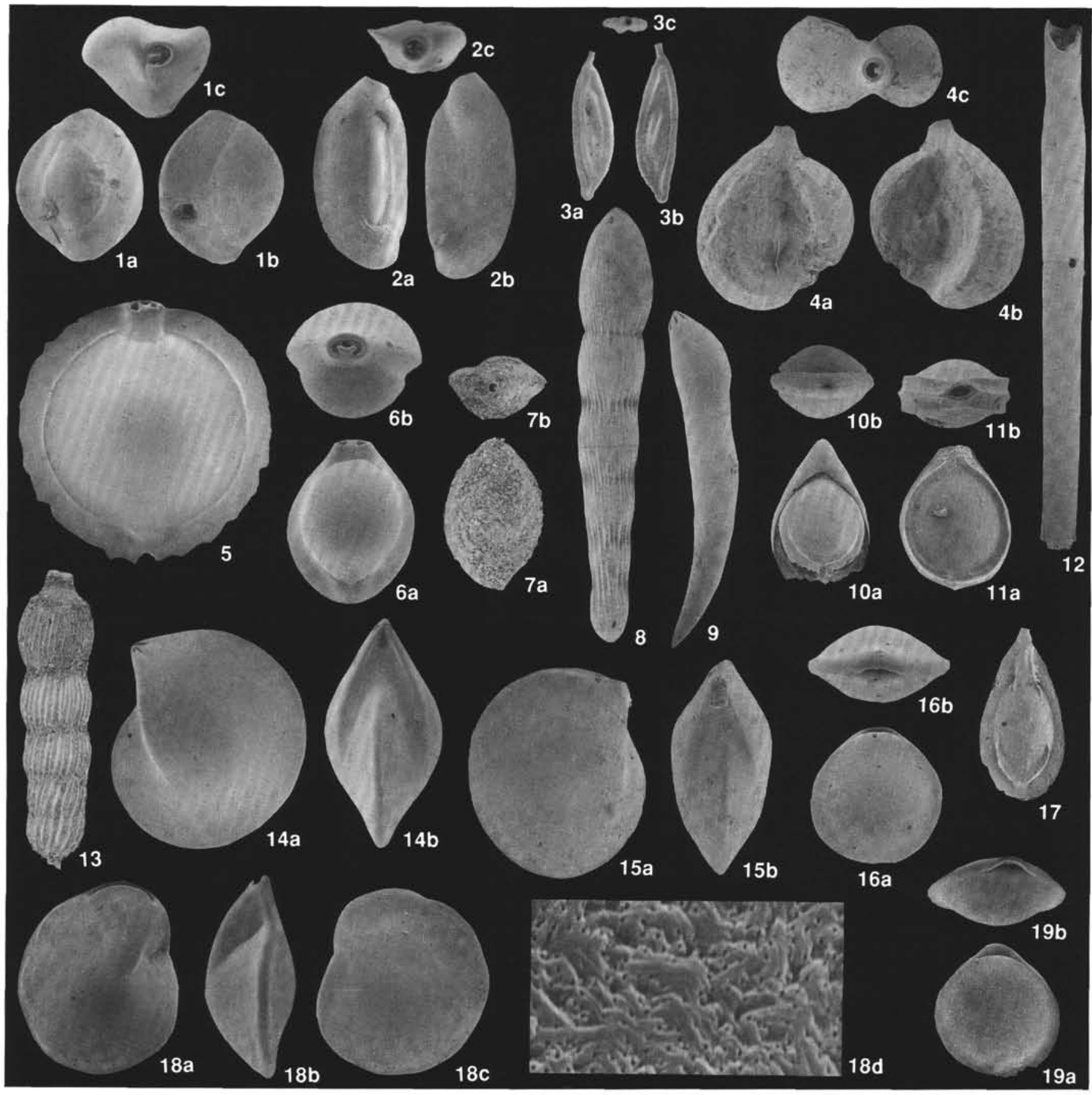

Plate 2. 1. Quinqueloculina sp. A; Sample 126-791B-23R-CC (21×). 2. Quinqueloculina lamarckiana d'Orbigny; Sample 126-793A-7H-3, 60-65 cm (46×). 3. Ophthalmidium pusillum (Earland); Sample 126-793A-3H-4, 0-1 cm (46x). 4. Quinqueloculina sp. B; Sample 126-792A-8H-1, 93-97 cm (21×). 5. Pyrgo murrhina (Schwager); Sample 126-790C-10X-CC (21×). 6. Pyrgo lucernula (Schwager); Sample 126-791B-22R-CC (32×). 7. Sigmoilopsis schlumbergeri (Silvestri); Sample 126-790C-19X-CC (21×). 8. Chrysalogonium longicostatum Cushman and Jarvis sp. A; Sample 126-793B-8R-CC (21×). 9. Dentalina communis d'Orbigny; Sample 126-792E-1R-1, 11-13 cm (21×). 10. Fissurina sp. A; Sample 126-790C-18X-CC (32×). 11. Fissurina bradyi Silvestri; Sample 126-790C-11X-CC (70x). 12. Nodosaria longiscata d'Orbigny; Sample 126-792E-35R-CC (21×). 13. Amphicoryna scalaris (Batsch); Sample 126-792E-3R-1, 64-69 cm (21×). 14. Lenticulina rotulatus (Lamarck); Sample 126-792A-5H-CC (32×). 15. Lenticulina sp. A; Sample 126-792E-5R-CC (32×). 16. Parafissurina sp. A; Sample 126-790C-12X-CC (32x). 17. Fissurina subformosa Parr; Sample 126-790C-20X-2, 36-41 cm (70x). 18. Hoeglundina elegans (d’Orbigny); Sample 126-790C-10X-CC (18a-c, 32× 18d, 4648×). 19. Parafissurina sp. B; Sample 126-790C-11X-CC (70×). 


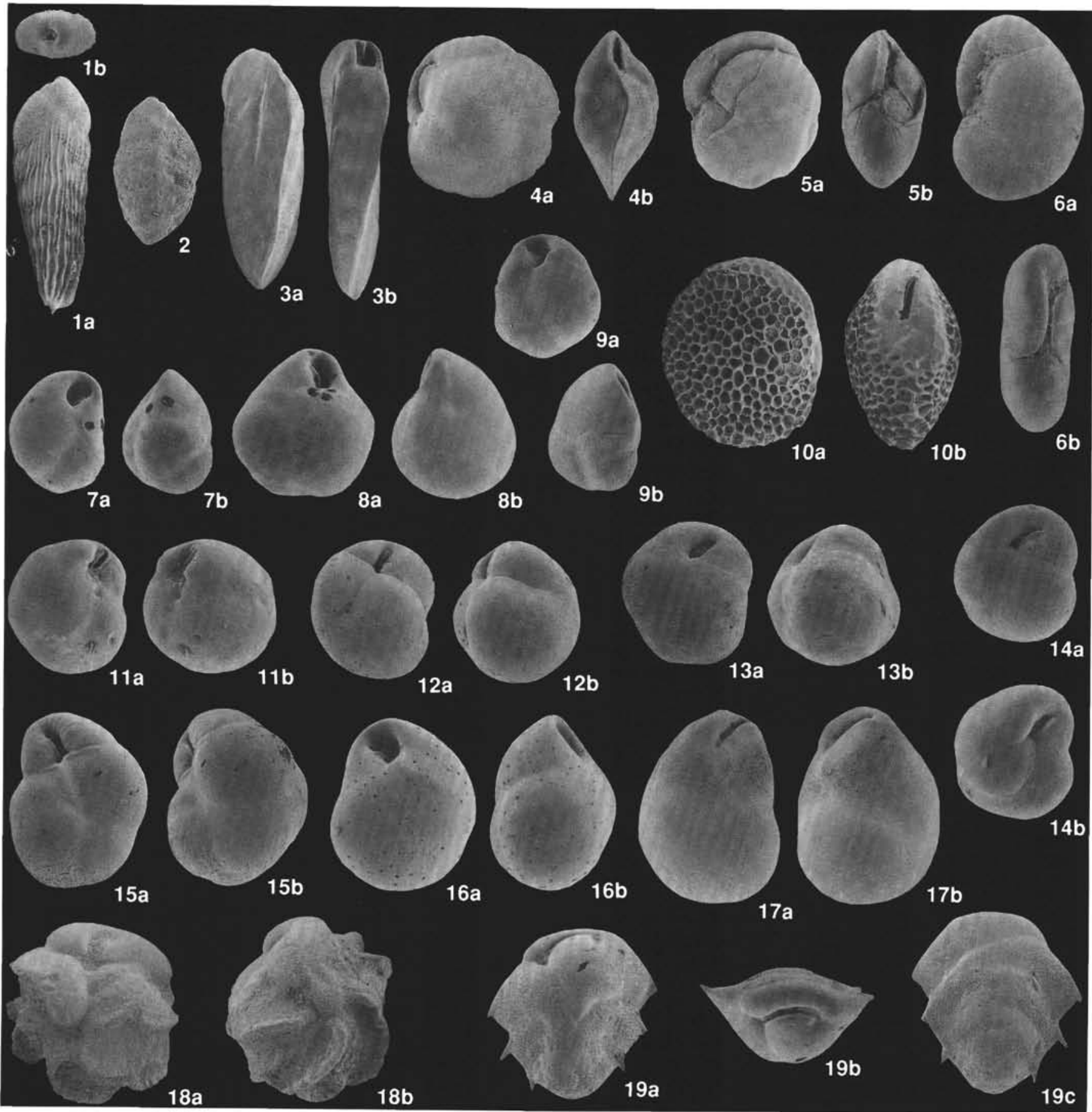

Plate 3. 1. Bolivina pusilla Schwager; Sample 126-790C-12X-CC (71×). 2. Bolivina robusta Brady; Sample 126-791B-41R-CC (47x). 3. Bolivinita quadrilat era (Schwager); Sample 126-791B-28R-CC (30x). 4. Cassidulina carinata Silvestri; Sample 126-790B-8H-6, 29-31 cm (95x). 5. Cassidulina sp. A; Sample 126-790C-20X-CC (71×). 6. Cassidulina sp. B; Sample 126-788D-4R-CC (95×). 7-9, 16. Globocassidulina moluccensis (Germeraad); (7-8) Sample 126793A-9H-CC (47x); $(9,16)$ Sample 126-791B-24R-CC (47x). 10. Favocassidulina favus (Brady); Sample 126-793A-9H-CC (33x). 11-15. Globocassidulina subglobosa Brady; (11) Sample 126-790C-19X-CC (47x); (12) Sample 126-792E-23R-1, 59-61 cm (47x); (13) Sample 126-793B-8R-CC (33x); (14) Sample 126-793B-2R-1, 36-38 cm (47x); (15) Sample 126-790C-12X-CC (33×). 17. Globocassidulina sp. A; Sample 126-790C-12X-CC (22x). 18. Islandiella elegans (Sidebottom). Sample 126-790C-20X-2, 36-41 cm (71×). 19. Ehrenbergina pacifica Cushman; Sample 126-790C-19X-CC (71×). 


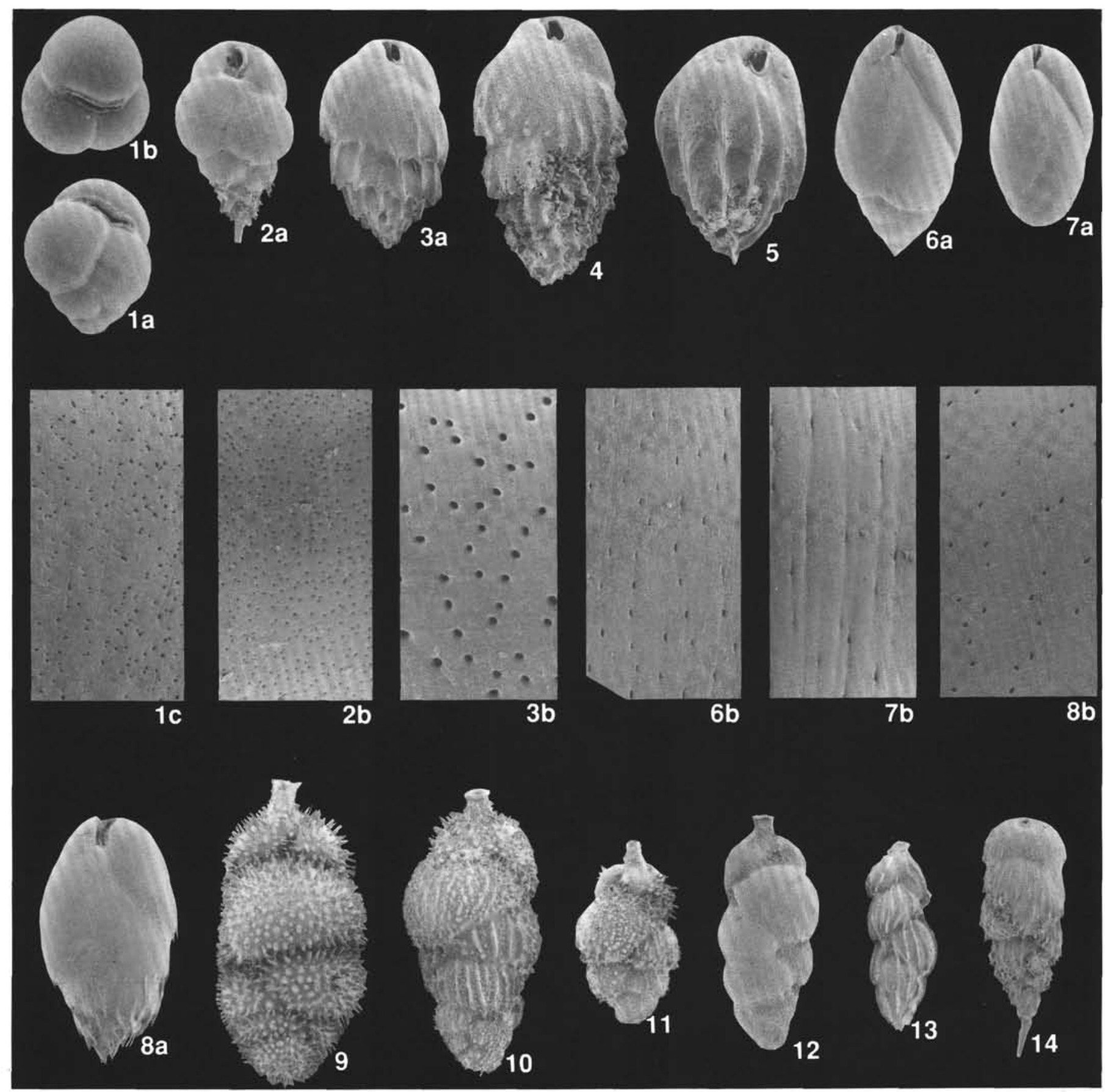

Plate 4. 1. Tosaia hanzawai Takayanagi; Sample 126-791A-31X-CC (1a-b, 47x 1c, 657×). 2. Bulimina aculeata d'Orbigny; Sample 126-790C-11X-CC (2a, $47 \times 2 b, 978 \times$ ). 3-4. Bulimina striata d'Orbigny; (3) Sample 126-791A-39X-1, 28-32 cm (3a, 47× 3b, 657×); (4) Sample 126-792B-8X-CC (47x). 5. Bulimina rostrata Brady; Sample 126-792E-15R-CC (98x). 6-7. Globobulimina affinis (d'Orbigny); Sample 126-790C-10X-CC (6a and 7a, 30x 6b and 7b, 657x). 8. Bulimina barbata Cushman; Sample 126-790C-14X-2, 61-63 cm (8a, 47x 8b, 657x). 9. Uvigerina hispida Schwager, Sample 126-790C-13X-CC (47x). 10-11. Uvigerina hispidocostata Cushman and Todd; (10) Sample 126-791B-10R-CC (47x); (11) Sample 126-788D-6R-CC (47x). 12. Uvigerina senticosa Cushman; Sample 126-790C-14X-2,61-63 cm (47x). 13. Uvigerina graciliformis Papp and Turnovsky; Sample 126-792E-15R-CC (47x). 14. Rectuvigerina multicostata (Cushman and Jarvis); Sample 126-792E-23R-1, 59-61 cm (47x). 


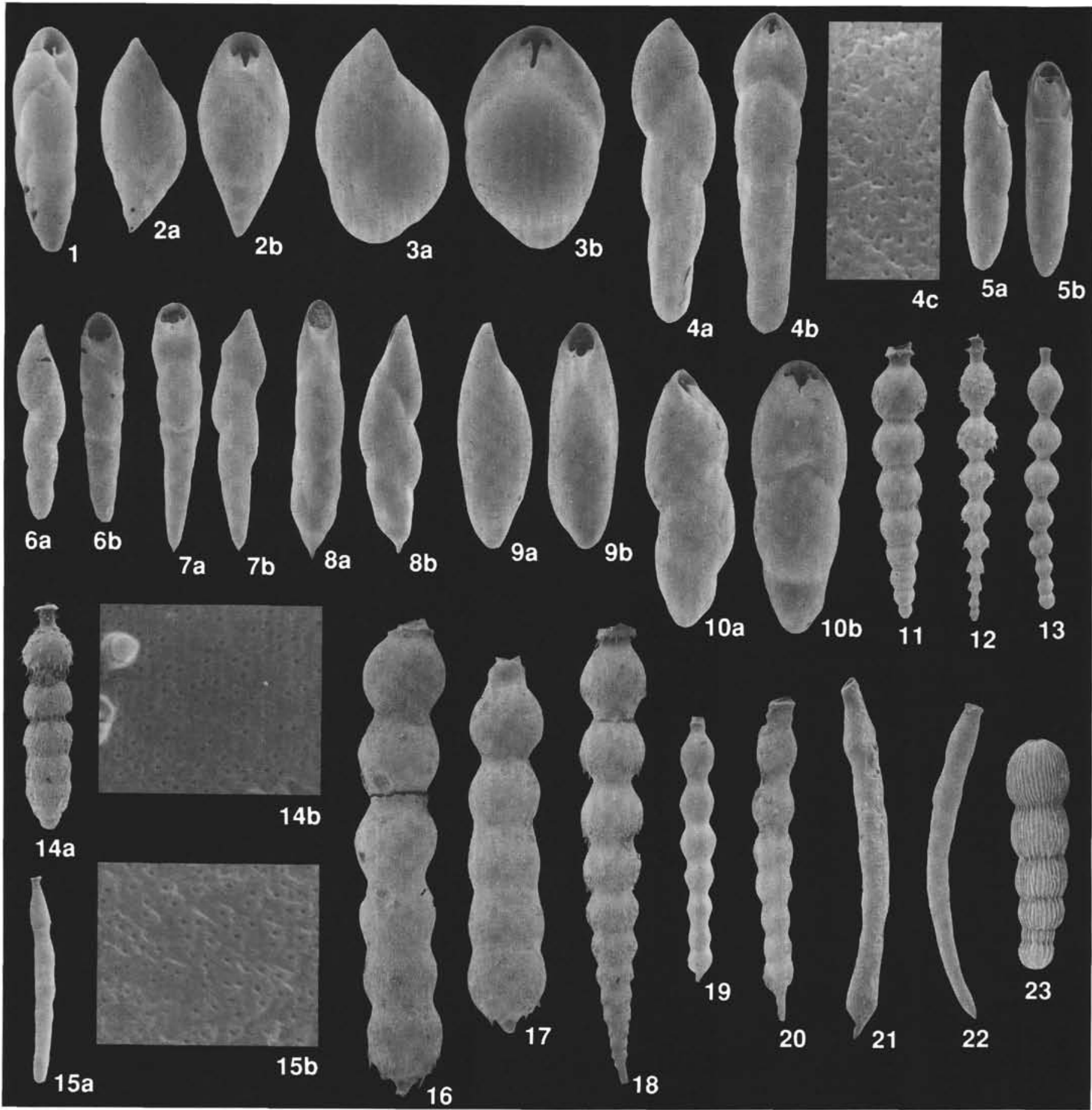

Plate 5. 1. Bulimina exilis Brady; Sample 126-790C-13X-CC (66x). 2. Pleurostomella praegerontica Cushman and Stainforth; Sample 126-792E-2R-CC (66x). 3. Pleurostomella brevis Schwager; Sample 126-792E-4R-1, 9-11 cm (46x). 4. Pleurostomella sp. A; Sample 126-791B-46R-CC (4a-b, 30x 4c, 4510x). 5. Pleurostomella sp. B; Sample 126-792E-2R-CC (66×). 6. Pleurostomella subcylindrica (Cushman); Sample 126-793B-17R-4, 58-60 cm (66×). 7. Pleurostomella sp. C; Sample 126-792A-8H-1,93-97 cm (30×). 8. Pleurostomella sp. D; Sample 126-790C-20X-2, 36-41 cm (66×). 9. Pleurostomella sp. E; Sample 126-791B-46R-CC (66×). 10. Pleurostomella acuta Hantken; Sample 126-793B-9R-1, 60-61 cm (66x). 11. Stilostomella sp. A; Sample 126-792E-3R-CC (46x). 12. Stilostomella lepidula (Schwager); Sample 126-790C-18X-CC (30×). 13. Stilostomella gracillima (Cushman and Jarvis); Sample 126-793B-8R-CC (46x). 14. Stilostomella sp. B = Orthomorphina aff. O. antillea (Cushman) of Boltovskoy, 1980; Sample 126-792E-6R-1, 58-60 cm (14a, 66x 14b, 6443x). 15. Stilostomella cf. S. annulifera (Cushman and Bermudez); Sample 126-792E-4R-1, 9-11 cm (15a, 46x 15b, 6443×). 16-17. Stilostomella abyssorum (Brady); Sample 126-793B-16R-CC (30x). 18. Stilostomella sp. C; Sample 126-792E-1R-1, 11-13 cm (30x). 19-20. Stilostomella sp. D; (19) Sample 126-792E-3R-CC (30x); (20) Sample 126-788C-28R-CC (30x). 21. Stilostomella sp. E; Sample 126-792E-57R-1, 97-99 cm (30×). 22. Stilostomella sp. F; Sample 126-792E4R-1, 9-11 cm (30x). 23. Stilostomella sp. G; Sample 126-792B-8X-3, 67-72 cm (46x). 

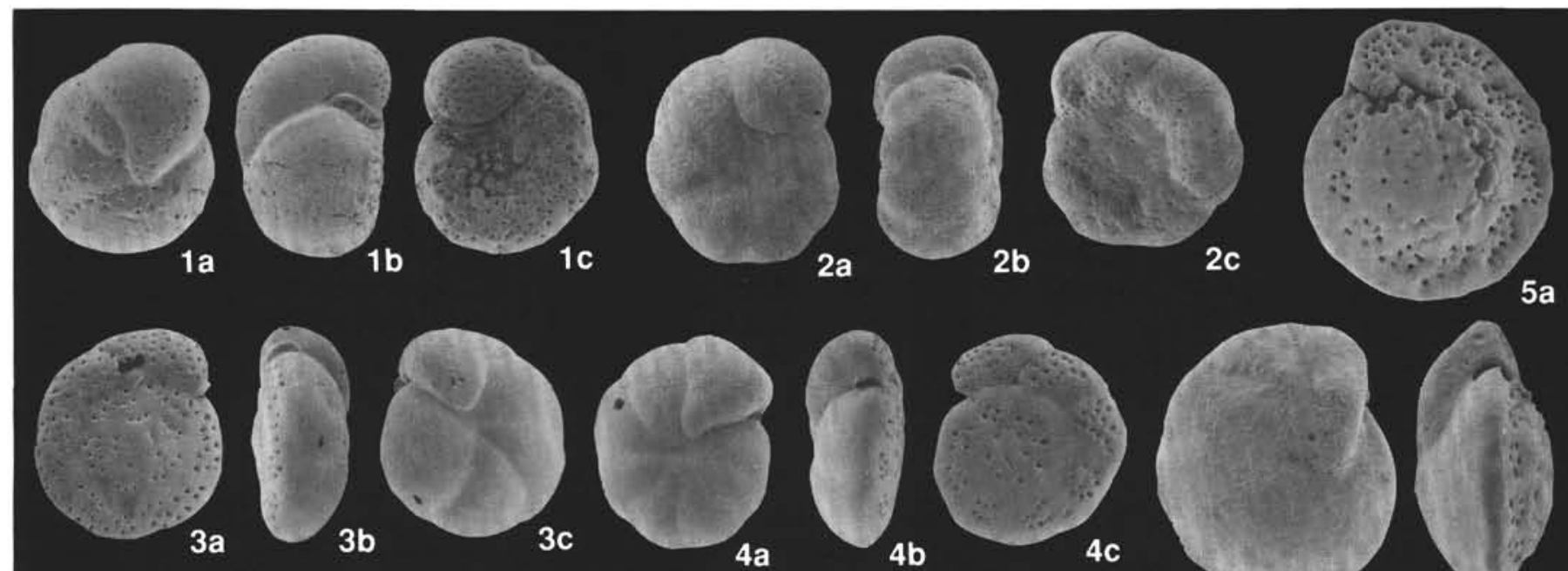

$4 b$
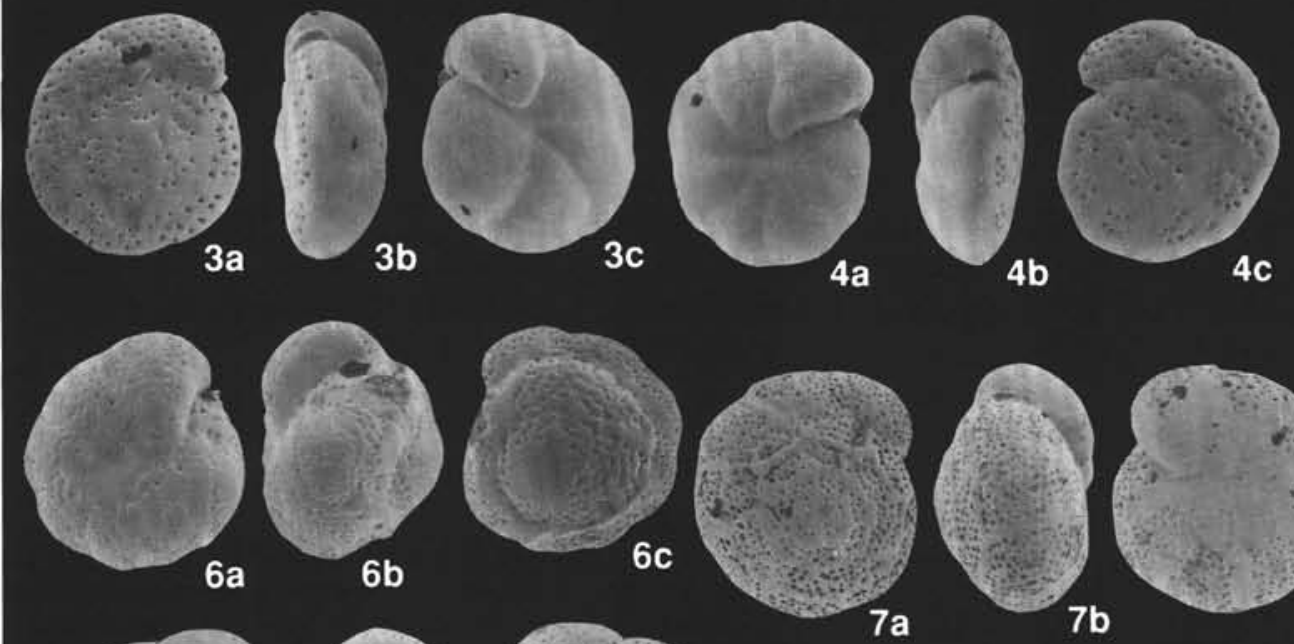

$5 b$

$5 c$
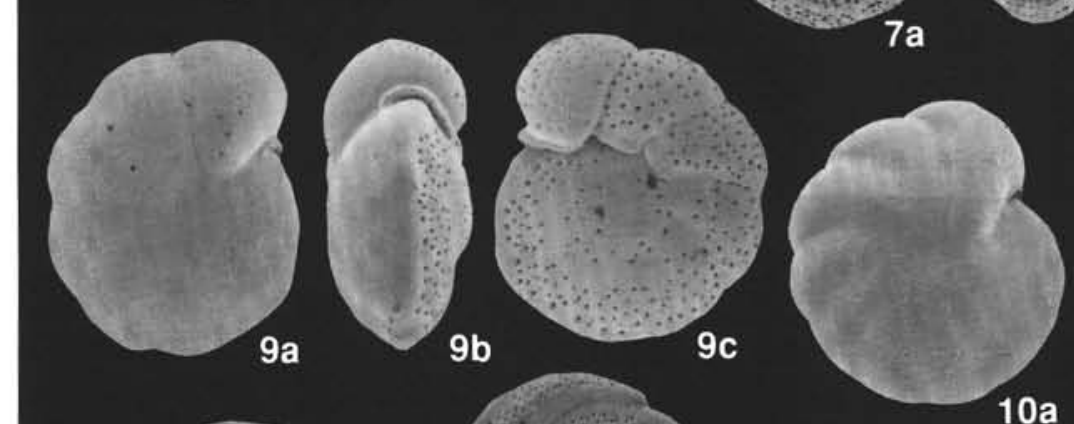

$7 \mathbf{b}$

$8 \mathrm{a}$

$8 b$

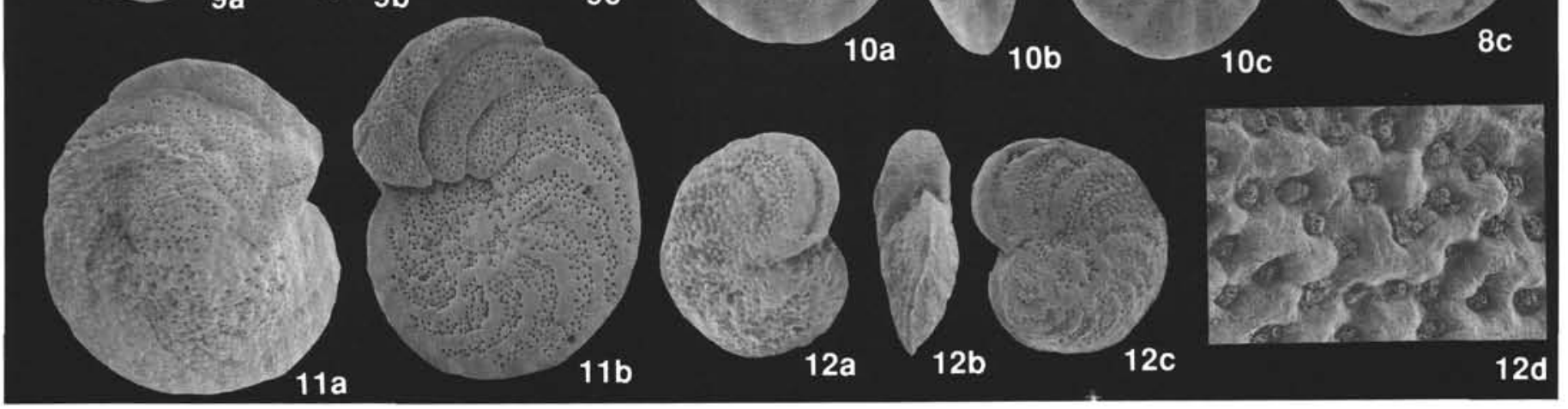

Plate 6. 1. Anomalinoides globulosus (Chapman and Parr); Sample 126-793B-11R-4, 88-90 cm (53×). 2. Cibicidoides sp. A; Sample 126-793B-8R-CC (53×). 3-4. Cibicidoides bradyi (Trauth); (3) Sample 126-790B-3H, 0-1 cm (53x); (4) Sample 126-790C-18X-CC (53x). 5. Cibicidoides mundulus (Brady, Parker, and Jones); Sample 126-790C-12X-CC (37x). 6-7. Cibicidoides bradyi (Trauth); (6) Sample 126-791A-31X-CC (37x); (7) Sample 126-792E-1R-1, 11-13 cm (37x). 8. Cibicidoides havanensis (Cushman and Bermudez); Sample 126-793B-16R-CC (24×). 9. Cibicidoides sp. B; Sample 126-790C-14X-2, 61-63 cm (53x). 10. Cibicidoides sp. C; Sample 126-793A-3H-4, 0-1 cm (106×). 11. Cibicidoides wuellerstorfi (Schwager); Sample 126-792A-3H-3, 59-64 cm (24×). 12. Cibicidoides renzi (Cushman and Stainforth); Sample 126-792E-23R-1, 59-61 cm (12a-c, 24× 12d, 238×). 

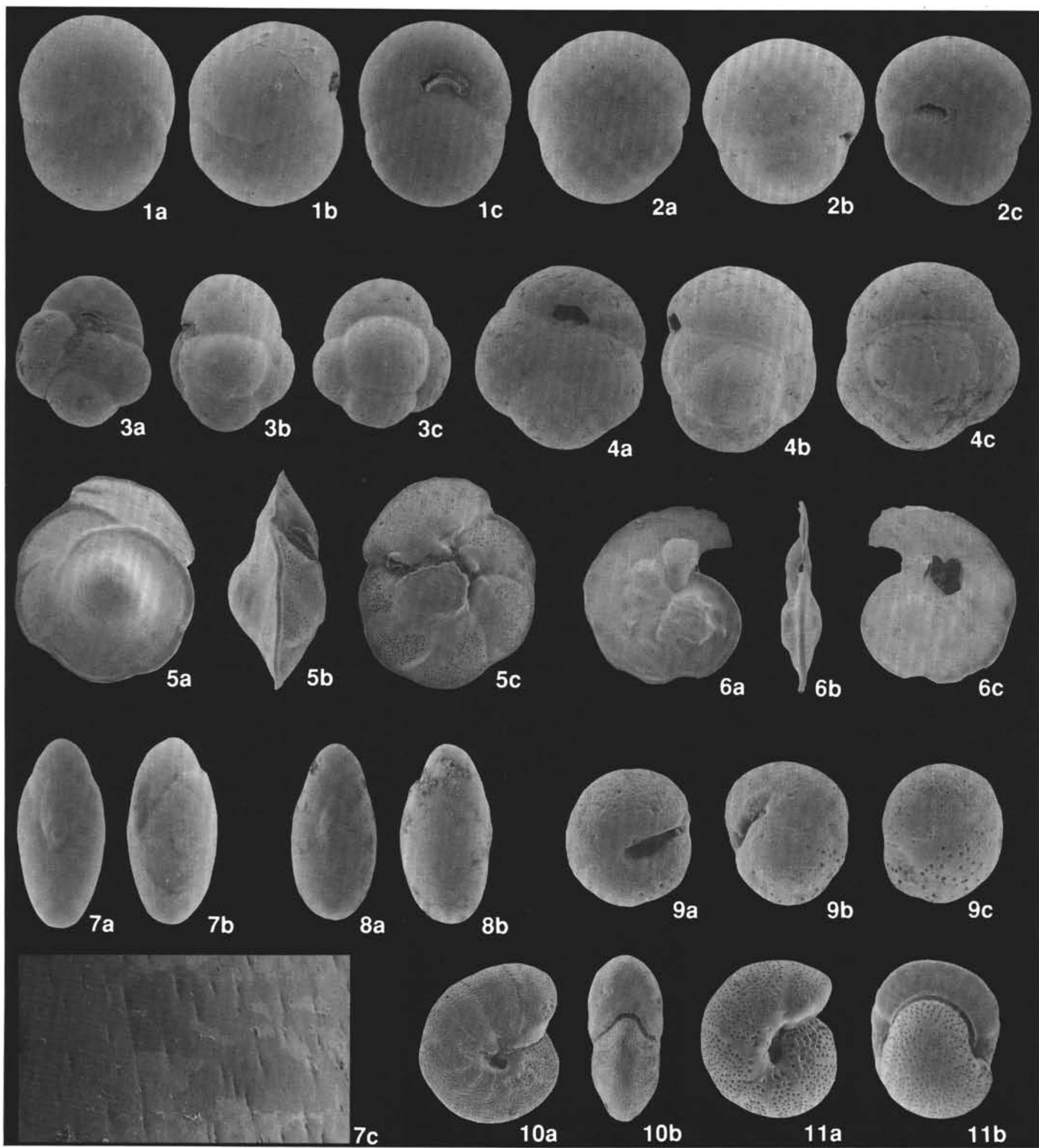

Plate 7. 1. Sphaeroidina sp. A; Sample 126-793A-5H-CC (44x). 2. Sphaeroidina bulloides d'Orbigny; Sample 126-790C-12X-CC (44x). 3-4. Sphaeroidina sp. B; (3) Sample 126-790C-20X-2, 36-41 cm (44x); (4) Sample 126-792E-16R-CC (44x). 5. Gavelinopsis lobatulus (Parr); Sample 126-790C-13X-CC (124X). 6. Laticarinina pauperata (Parker and Jones); Sample 126-793A-7H-3, 60-65 cm (28x). 7-8. Chilostomella oolina Schwager; (7) Sample 126-790A-2H-3, 98-100 cm (7a-b, 44x; 7c, 930x); (8) Sample 126-788C-28R-CC (44x). 9. Saitoella globosa Kaiho, gen. nov. et sp. nov.; Sample 126-792E-32R-1, 13-15 cm (63); (9a, ventral view; 9b, edge view; 9c, dorsal view). 10. Melonis barleeanus (Williamson); Sample 126-790C-12X-CC (44X). 11. Melonis pompilioides (Fichtel and Moll); Sample 126-790C-10X-CC (44x). 

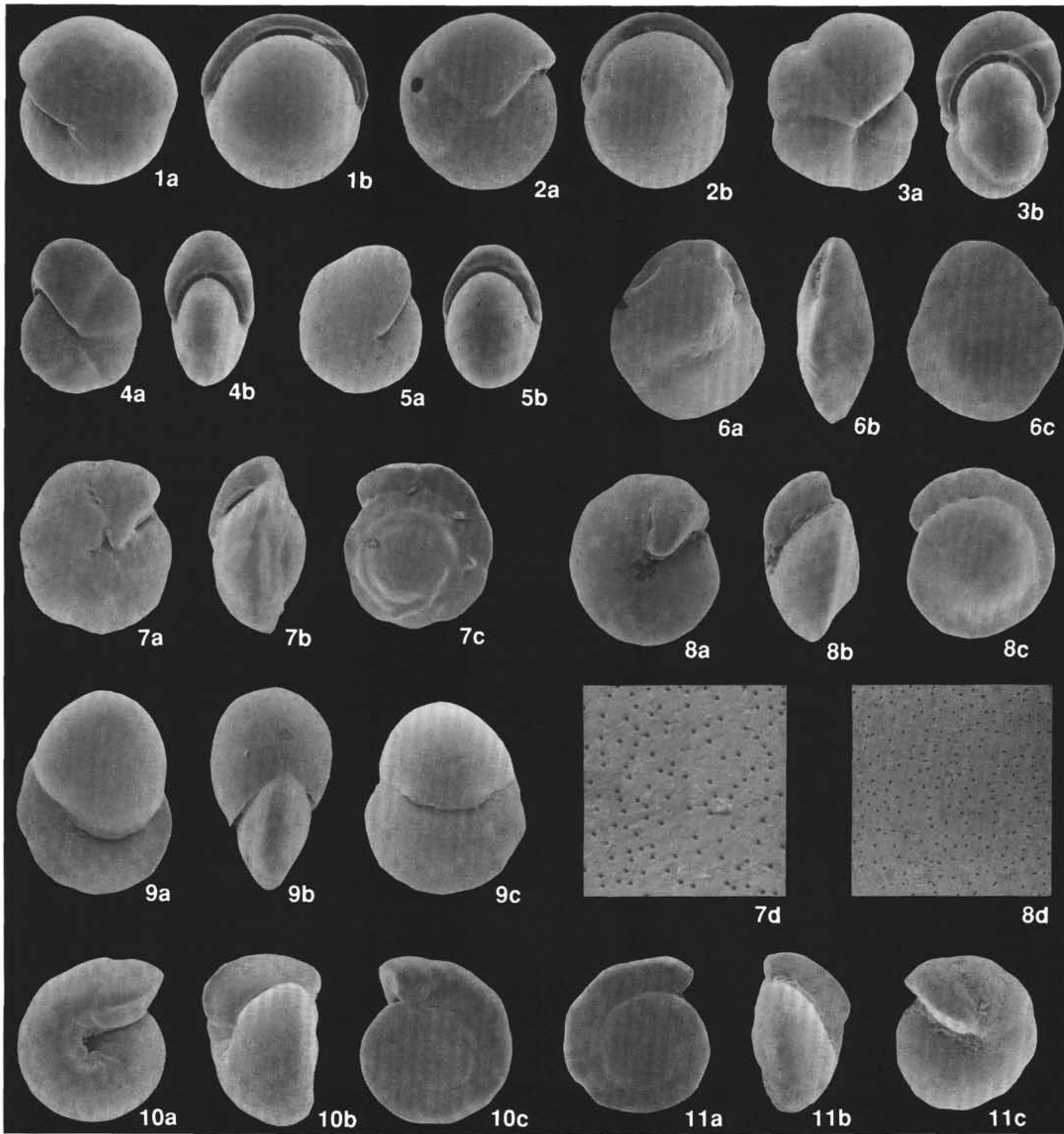

Plate 8. 1-2. Pullenia bulloides (d'Orbigny); (1) Sample 126-790C-10X-CC (59x); (2) Sample 126-790C-18X-CC (88x). 3. Pullenia quinqueloba (Reuss); Sample 126-790C-13X-CC (59x). 4. Pullenia simplex Rhumbler; Sample 126-790A-2H-3, 98-100 cm (59x). 5. Pullenia riveroi Bermudez; Sample 126-790C10X-CC (59x). 6. Epistominella exigua (Brady); Sample 126-793A-5H-CC (88x). 7. Oridorsalis umbonatus (Reuss); Sample 126-790C-10X-CC (7a-c, 26x 7d, 1765×). 8. Gyroidinoides sp. A; Sample 126-791B-28R-CC (8a-c, 41× 8d, 883×). 9. Globogyroidina boninensis Kaiho, gen. nov. et sp. nov.; Sample 126-790A-2H-3, 98-100 cm (59x); (9a, ventral view; 9b, edge view; 9c, dorsal view). 10. Gyroidinoides neosoldanii Brotzen; Sample 126-792A-10H-3, 62-66 cm (26x). 11. Gyroidinoides sp. B; Sample 126-788D-4R-CC (59x). 